\title{
Hybrid optimizers to solve a tri-level programming model for a tire closed-loop supply chain network design problem
}

Amir Mohammad Fathollahi-Fard ${ }^{a^{*}}$, Mostafa Hajiaghaei-Keshteli ${ }^{\mathrm{a}}$, Seyedali Mirjalili ${ }^{\mathrm{b}}$

${ }^{a}$ Department of Industrial Engineering, University of Science and Technology of Mazandaran, Behshahr, Iran

${ }^{b}$ Institute for Integrated and Intelligent Systems, Griffith University, Nathan, QLD 4111, Australia

* Corresponding author

E-mail addresses: amirfard@mazust.ac.ir (A. M. Fathollahi-Fard), mostafahaji@mazust.ac.ir (M. Hajiaghaei-Keshteli), seyedali.mirjalili@griffithuni.edu.au (S. Mirjalili)

Graphical abstract

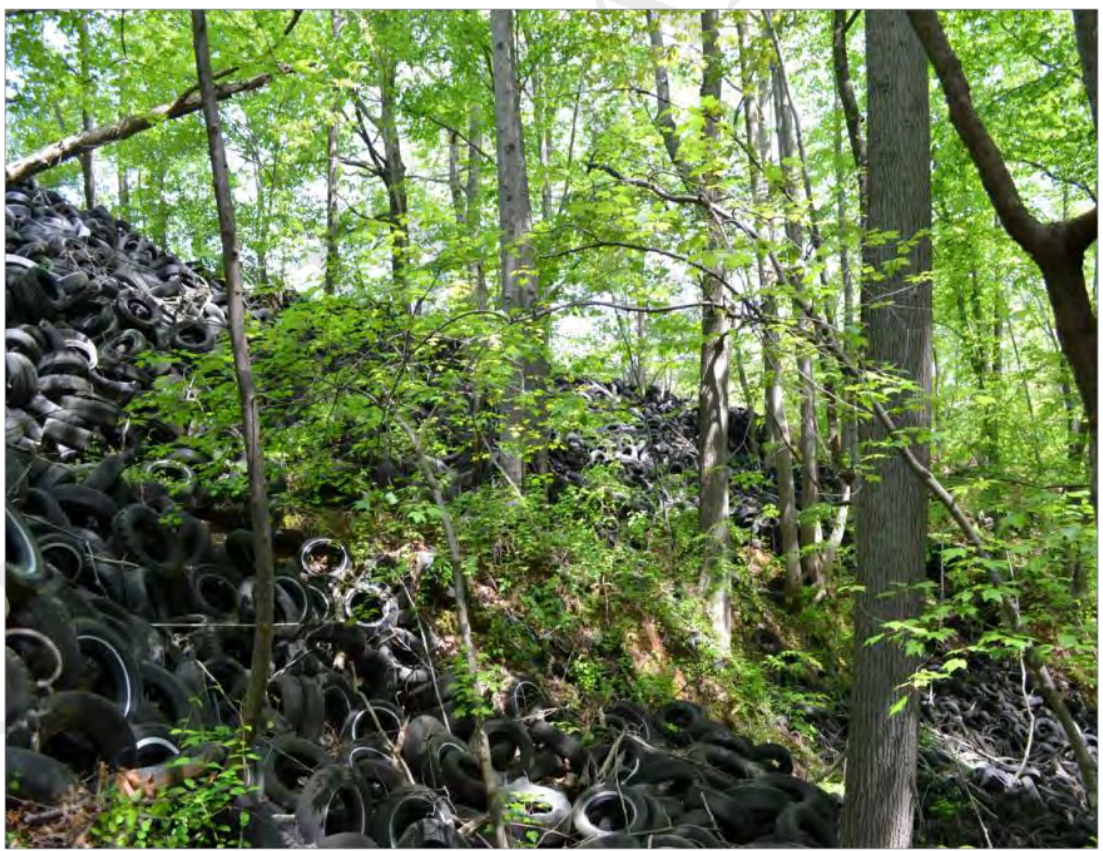

The scraped tires may be left in nature to pollute the environment (this photo is taken from our case study) 


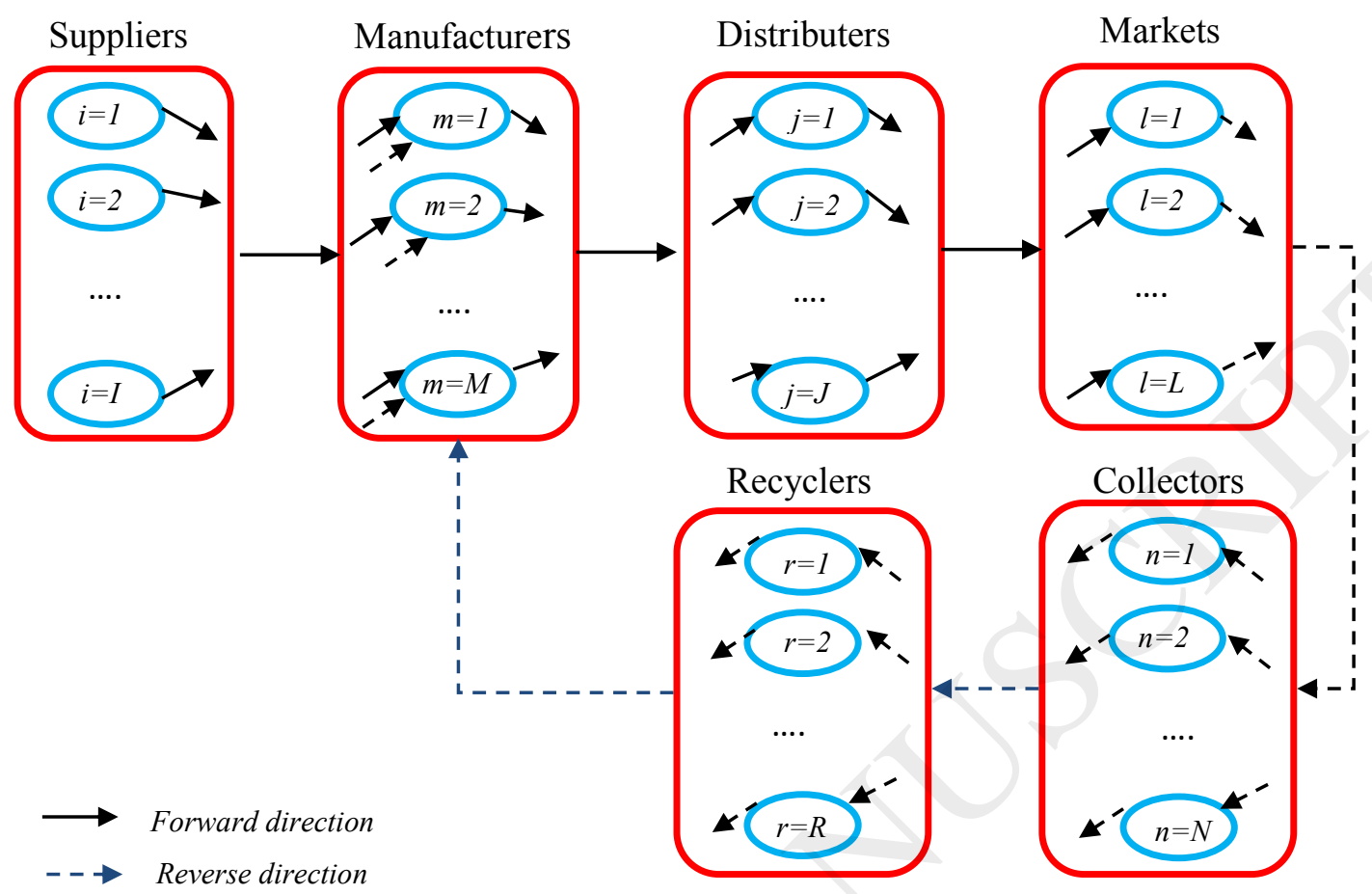

The graphical structure of tire closed-loop supply chain

\section{Highlights}

- Designing a tri-level programming model for the tire closed-loop supply chain;

- Proposing four new hybrid optimizers to improve the recent and old used meta-heuristics in an efficient way;

- Comparing the hybrid algorithms with their general ideas by a set of comprehensive analyses;

- Demonstrating the benefits of the proposed methodology using a real case study and sensitivity analyses;

- Among these hybrid methods, HKASA shows the best performance in practice.

\section{Abstract}

The Closed-loop Supply Chain (CLSC) is one way to collect and recycle scraped tires. CLSC consists of a set of suppliers, manufacturers, distributors and customers in a forward system. Collectors and recyclers are formed a reverse one as well. The decisions in such systems are adopted in a hierarchy. 
Regarding this issue, this study develops a tri-level programming model to design the location-allocation of the tire CLSC for the first time. The proposed model is formulated on the static Stackelberg game between manufacturers, distributors and collectors in the framework of CLSC. The performance of the current exact solutions for this problem suffers from degraded performance when solving large-scale problems. To alleviate this drawback, this study proposes a number of new hybrid optimizers by considering the benefits of recent metaheuristics. An extensive comparative study confirms the efficiency of developed model and the performance of the hybrid optimizers proposed when solving the large-scale problems.

Keywords: hybrid optimizers; metaheuristic algorithms; tri-level programming; location-allocation problem; tire closed-loop supply chain;

\section{Introduction and literature review}

In the recent years, there has been an increasing interest in terms of Closed-Loop Supply Chain (CLSC). The profits of collecting and recycling the products lead to investigate the Reverse Logistic (RL) and CLSC systems [1]. The main operation in the CLSC is considered as the location of facilities and allocation of different parts of a system [2]. In such systems, the management is determined by different levels (e.g. manufacturers and distributors in the forward system and collectors in the reverse ones) [3]. All parts of a supply chain act in a cooperative manner to decide the best strategy [4]. The behavior of each level is adopted according to its benefit and the best strategy should be selected [5]. In this way, the multi-level programming is an efficient tool to simulate such systems [6]. In this work, a tri-level location-allocation model is designed for tire CLSC. Accordingly, the manufacturers are the first level, distributers form the second level and collectors guaranty the returned scraped tires in the reverse loop as the third level. The motivation of presented tire closed-loop supply chain is addressed by the following sub-section.

\subsection{Motivation}

There are more than one billion brands for tire in the world with nearly 500 producers across the globe [7]. According to the report of the U.S. Environmental Protection Agency (EPA), each U.S. family has three cars that used 24 tires every 3 years. As such, approximately 290 million scraped tires are disposed every year of which $20 \%$ are illegally scraped in landfills or roadsides. 
This causes an important disposal problem with health hazard and environmental damages [8]; [9]. In addition, the chemicals leached from the scraped tires are hazardous for human health, water and air pollution [7]. Unfortunately, in major cases, the scraped tires are released in the nature without considering the affection on environments. Figure 1 shows an example, in which thousands of scraped tires contaminate the nature of jungles in northern regions of Iran.

Tire recycling is a process of converting scraped tires into material that can be utilized again for manufacturing [10]. Tire recycling has gained much attention in recent years by government across the globe. For instance, Chopra and Meindle [11] discussed in 2015 that in U.S. only three percent of scraped tires are recycling between 2009 and 2011. Overall, it shows the importance of recycling for the scraped tires.

\subsection{Tire closed-loop supply chain}

In the literature of CLSC and RL methodologies, there are several articles in this area of which only a few papers review the related studies and suggest future works (See [12] [13] [14] [15]).

In the area of tire supply chain, less than ten studies have been published despite its importance. In 1997, Ferrer [16] estimated the optimal number of times which the tires can be reused. In 2009, the mathematical model to probe the tire supply chain was introduced by Kannan et al. [17]. They developed a CLSC system for tires and plastic via a Mixed Integer Linear Programming (MILP). In addition, an RL system for the tire supply chain was addressed by Sasikumar et al. [18] in 2010 for truck tire remanufacturing process. In another seminal research, Subulan et al. [7] introduced a case study in Turkey to investigate this issue. They used a Fuzzy Mixed Integer Programming (FMIP) by a fuzzy solution approach to address their CLSC optimization model. In the top of the most recent works, Amin et al. [10] developed an economic optimization model for tire CLSC under uncertainty conditions in Toronto, Canada. In this paper, a tri-level programming model was used to simulate the decision levels for tire CLSC by a real data set.

Overall, it is evident that the application of such models should be examined in a real case study to achieve the managerial implications of tire supply chain. This reason motivated several works including the current work to use a real case study. Another main similarity of mentioned studies is referred to considering the RL operations in an efficient way to ease the managing of such systems [2]. Unlike the past studies, this study proposes a tri-level programming model to 
reach this goal more efficiently. It has been proven that a multi-level programming gives a performance tool to manipulate the RL and CLSC operations [19].

In our proposed system, manufacturers can purchase raw material from suppliers and recyclers. Distributers as the middle-level buy the different types of tires from manufacturers and sell them to markets. Finally, collectors as the bottom-level collect the scraped tires from customers and prepare them for recycling centers. Figure 2 shows the graphical structure of the proposed tire CLSC model.

\subsection{Multi-level programming for location-allocation problem}

Multi-level programming or multi-level decision-making is a kind of hierarchical optimization problem. This type of optimization model is used in many applications especially for locationallocation problems [19]. The simplest case of multi-level programming is bi-level. In this method, the objective function of lower-level forms a constraint of upper-level model. The bilevel programming is formulated on the game theory of Stackelberg [20]. This type of model was presented by Bracken and McGill [21] for the first time. They defined the bi-level programming as a "mathematical program with optimization in the constraint". As pointed out by Ben-Ayed [22], bi-level models are difficult to solve in any sizes. The easiest case of a bi-level model is obviously hard and known as NP-hard. Due to the degraded performance of exact methods when solving this NP-hard problem, various heuristics and metaheuristics have been developed in the literature [5].

Up to our knowledge, the location-allocation problem was first modeled by Taniguchi et al. [23] using bi-level programming. They proposed a capacitated location-allocation of crossdocking system. A Genetic Algorithm (GA) was employed in their work to solve the non-linear upper level model. Huang and Liu [24] also used GA to solve the two-stage supply chain model with balanced workload requirement for the lower level. Generally, regarding the literature, there are also some other works utilizing the multi-level programming to formulate the locationallocation problem. For instance, a hybrid of GA and Particle Swarm Optimization (PSO) was considered by Kuo and Han [25] to solve the underlying bi-level location-allocation model. Ma et al. [26] developed a bi-level model in which the upper level was manufacturers and the retailers formed the lower-level problem. They also used PSO to solve their model. In 2014, Zhang and $\mathrm{Xu}[27]$ added the demand uncertainty and continued cost function for the lower-level 
in their proposed bi-level programming model. They employed a heuristic-based GA to address the model. In 2016, one of interesting works in the literature was conducted by Saranwong and Likasiri [28], in which the location-location problem, which considered the distributers as the upper-level and customers in the lower-level, was proposed. They solved the model with five heuristic algorithms and verified the performance of model through a real case study. In a similar study, the same authors explored the Thailand's sugarcane management by a bi-level programming approach [29].

In 2017, Parvasi et al. [30] proposed a bi-level programming model for a location-allocationrouting problem for content of public transportation systems with possibility of outsourcing demand. They also used GA and SA to solve their bi-level model. Recently, Fathollahi Fard and Hajaghaei-Keshteli [19] proposed a tri-level location-allocation model for forward/reverse supply chain network design problem. They approved the application of their model in glass industry through a case study in Iran. They employed five metaheuristics including Tabu Search (TS), Variable Neighborhood Search (VNS), PSO, Keshtel Algorithm (KA) and Water Wave Optimization (WWO) to solve the problem.

Taken together, the mentioned studies revealed that metaheuristics are effective approaches to solve the underlying multi-level programming model for location and allocation problem. The literature showed that using hybrid algorithms is steel scarce in this area [4]. Additionally, the No Free Lunch theorem [31] states that there is no optimization algorithm to solve all optimization problems. Therefore, the current algorithms might need modifications, tunings, or hybridizations to solve a set of specific problems [32]. These drawbacks motivate us to attempt a set of new hybrid optimizers as the main contributions of this study.

\subsection{Highlights and contributions}

Generally speaking, the aforementioned studies in this area can be divided into three categories: improving optimization model, improving current solution approaches or suggesting new optimizers, and improving both model and solution algorithms. CLSC may be formulated and modeled with different assumptions and considering more factors [33]. Although adding more elements makes CLSC more practical, it increases the difficulty and computation cost of this problem [34]; [35]. This means that changing case model and/or case study will change the search space of the problem and might result in getting poor results using the current optimizers 
[36]. These facts keep this area active and motivate our attempts to contribute to both areas: a new optimization model along with optimizer improvement by four new hybrid metaheuristics.

To cope with the described problem as illustrated in the last three sub-sections, this study proposes a tri-level location-allocation model for tire closed-loop supply chain. One of the advantages of this technique is to decide the best strategy for the main level for location and allocation of facilities [4]. To the best of our knowledge, this study is the first attempt to consider a closed-loop supply chain for tire products. In 2018, Fathollahi Fard and Hajaghaei-Keshteli [19] proposed a tri-level programming model for a forward/reverse supply chain, but this study considers a tire CLSC for the first time by a tri-level programming approach. Another main contribution of this study in comparison with other similar papers is to propose four new hybrid optimizers based on recent and old metaheuristics. As mentioned earlier, in the developed model, manufacturers, distributors and collectors are formed the three main decision makers for the tire closed-loop supply chain which considers as the first, second and third levels of offered model, respectively. The main contributions of this study can be outlined as follows:

- Developing a tri-level programming model for tire closed-loop supply chain for the first time.

- Applying a nested approach, namely, tri-level metaheuristic, to solve the underlying trilevel model.

- Keshtel Algorithm (KA) [37] and Stochastic Fractal Search (SFS) [38] (as recent metaheuristics), Simulated Annealing (SA) [39] and Variable Neighborhood Search (VNS) [40] (as the conventional optimization algorithms, and four hybrid optimizers are evaluated.

- Comparing the results of the hybrid meta-heuristics with general ideas through different criteria in the terms of hitting time (the first time that algorithms find the best solution), solution quality, convergence of algorithms and time consumption.

- Validation of the proposed methodology by sensitivity analyses through a real case study.

The rest of the paper is organized as follows. Section 2 illustrates the problem description and model formulation. In Section 3, the solution approaches with illustration of proposed hybrid optimizers are given. In Section 4, the experimental results of algorithms are represented by a 
comprehensive evaluation in different criteria. Finally, the conclusion and future works are discussed in Section 5.

\section{Proposed problem}

In this section, we consider the multi-level tire closed-loop supply chain including suppliers, manufacturers, distributers, customers, collectors, and recyclers. A manufacturer supplies the raw material of tires from suppliers and recyclers. Then, manufactured tires are distributed in market by distributers. Collectors collect a part of the scraped tires that were used by customers in markets and send to recycling facilities. After recycling, the recycled material can be sent back to tire supply chain for remanufacturing. The CLSC problem covers both forward flow (i.e. from supplier to market) and reverse flow (i.e. from market to manufacturer or recycler). The tire closed-loop supply chain network is shown in Figure 2. A tri-level programming is employed to formulate the tire closed-loop supply chain. The structure of tri-level model is depicted in Figure 3. As can be seen, the manufacturers, distributors and collectors are the decision levels for the developed tri-level model. The suppliers, recyclers and markets are fixed. For each decision level, we want to optimize the location of facilities and allocation of products. In the next subsection, the characteristics of the problem are illustrated.

\subsection{Problem description}

This study aims to tackle the location-allocation problem for designing the tire closed-loop supply chain by considering three decision levels to select an optimized strategy for each level. The proposed problem is a multi-echelon, multi-product with multiple nodes among echelons as shown in Figure 2. Each facility of supply chain can be opened in a potential location with specific opening fixed cost $\left(F C_{\square}^{\square}\right)$, variable cost $\left(M C_{\square}^{\square}\right)$, holding cost $\left(H_{\square}^{\square}\right)$, purchasing cost ( $\left.P C_{\square}^{\square}\right)$, maximum number of each facility per level $\left(M A X_{\square}\right)$, storage capacity ( $\left.C A P_{\square}^{\square}\right)$, safety stock $\left(S_{\square}^{\square}\right)$ and transportation cost between other facilities $\left(T C_{\square}^{\square}\right)$. For example, the fixed, variable and holding costs for opening manufacturing plant in the potential location $m$ with a storage capacity $C A P_{m p}^{M}$ and safety stock $S_{m p}^{M}$ (regarding the type of tire $p$ ) are considered by $F C_{m}^{M}, M C_{m p}^{M}$ and $H_{m p}^{M}$ respectively. In addition, transportation costs have been considered by $T C_{i m}^{I}$ for transporting the raw materials between suppliers and manufacturers two different echelons. Similarly, the transportation cost of product $p$ between manufacturer plant $m$ and 
distributer center $j$ is $T C_{m j p}^{M}$. Manufacturer $(m)$ can supply the raw material from supplier $(i)$ or recycler $(r)$ with different price $P C_{i}^{I}$ or $P C_{r}^{R}$. The distributer $(j)$ purchase the tire type $p$ from the manufacturer $m$ with price $P C_{m p}^{M}$ and distribute in market $(l)$ with price $P C_{j p}^{J}$ according to the markets' demand $d_{l p}$ which has been generated by a normal distribution function $\left(N\left(d_{l p}^{\mu}, d_{l p}^{\delta}\right)\right)$. In the reverse flow, however, the potential collector center $(n)$ collect $\alpha_{l p}$ percentages of the scraped tires $(p)$ with price $P C_{l p}^{L}$ from market $(l)$. This fraction of returned products has been estimated by a normal distribution function $\left(\alpha_{l p} \sim N\left(\alpha_{l p}^{\mu}, \alpha_{l p}^{\delta}\right)\right)$. The potential recycler plant location $(r)$ purchases the scraped tire type $(p)$ from collector $(n)$ with price $\left(P C_{n p}^{N}\right)$ and sell to potential manufacturing/remanufacturing plant with price $P C_{r}^{R}$. Overall, the proposed model aims to determine the locations of the facilities and the right allocations in the forward and reverse flows of tire closed-loop supply chain by considering a tri-level programming model as the framework.

\subsection{Assumptions}

The problem investigated in this work is addressed considering the following assumptions:

- This model considers a multi-product, single period, and multiple facilities in a closedloop supply chain.

- Only manufacturers, distributors and collectors can decide their strategies to be optimized. These levels are the decision levels of CLSC in the presented model. The rest of them are fixed.

- Regarding each decision level, the fixed and variable cost, transportation cost, production cost and holding cost are considered to be optimized.

- The demand of each costumer must be met.

- $\quad$ Each facility may be assigned to more than one facility.

- The location of facilities should be determined regarding the optimal solution in each level.

- There is no flow between the same facilities in each level.

- The capacity of each facility in each location is limited and related to the demand. 
- As a supposition assumption from Devika et al. [1], a part of distributed tires in markets must be collected after utilization as scrapped tires.

- To consider the uncertainty of demand of markets along with the quantity of returned tires, a normal distribution function is used to estimate them [14].

- The raw material price of the recyclers is less than supplier.

\subsection{Notations}

The indices, parameters and variables used to mathematically formulate the problem are described below.

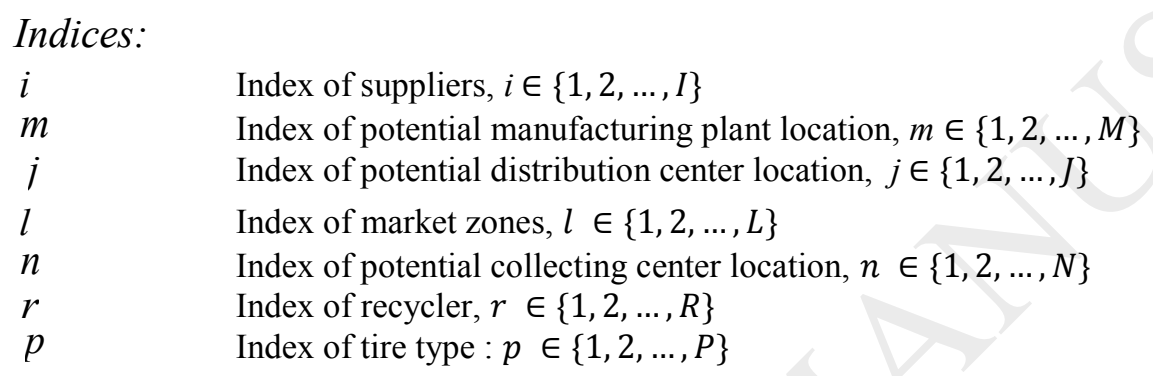

Parameters:

$T C_{i m}^{I}$

$T C_{m j p}^{M}$

$T C_{j l p}^{J}$

$T C_{\ln p}^{L}$

$T C_{n r p}^{N}$

$T C_{r m}^{R}$

$F C_{m}^{M}$

$F C_{j}^{J}$

$F C_{n}^{N}$

$M C_{m p}^{M}$

$M C_{j p}^{J}$

$M C_{n p}^{N}$

$P C_{i}^{I}$

$P C_{m p}^{M}$

$P C_{l p}^{L}$

$P C_{j p}^{J}$
Transportation cost per unit form supplier $i$ and manufacturer $m$

Transportation cost per unit for tire type $p$ from manufacturer $m$ and distributer $j$

Transportation cost per unit for tire type $p$ from distributer $j$ and market $l$

Transportation cost per unit for tire type $p$ form market $l$ and collector $n$

Transportation cost per unit for tire type $p$ form collector $n$ and recycler $r$

Transportation cost per unit form recycler $r$ and manufacturer $m$

Fixed opening cost of manufacturer $m$ to be established

Fixed opening cost of distributer $j$ to be established

Fixed opening cost of collector $n$ to be established

Variable manufacturing cost per unit for tire type $p$ and manufacturer $m$

Variable distributing cost per unit for tire type $p$ and distributer $j$

Variable collecting cost per unit for tire type $p$ and collector $n$

Cost of purchasing raw material for all types of tires for supplier $i$

Cost of purchasing tire type $p$ for manufacturer $m$

Cost of purchasing tire type $p$ for market $l$

Cost of purchasing tire type $p$ for distributer $j$ 
$P C_{n p}^{N}$

$P C_{r}^{R}$

$H_{m p}^{M}$

$H_{n p}^{N}$

$H_{j p}^{J}$

$M A X_{m}$

$M A X_{j}$

$M A X_{n}$

$d_{l p} \sim N\left(d_{l p}^{\mu}, d_{l p}^{\delta}\right)$

$d_{l p}^{\mu}$

$d_{l p}^{\delta}$

$\alpha_{l p} \sim N\left(\alpha_{l p}^{\mu}, \alpha_{l p}^{\delta}\right)$

$\alpha_{l p}^{\mu}$

$\alpha_{l p}^{\delta}$

$C A P_{i}^{I}$

$C A P_{m p}^{M}$

$C A P_{j p}^{J}$

$C A P_{n p}^{N}$

$C A P_{r p}^{R}$

$S_{m p}^{M}$

$S_{n p}^{N}$

$S_{i}^{I}$

$S_{j p}^{J}$

$S_{r p}^{R}$

Decision variables:

$X_{\text {im }}^{I}$

$X_{r m}^{R}$

$X_{m j p}^{M}$

$X_{j l p}^{J}$

$X_{\ln p}^{L}$

$X_{n r p}^{N}$

$Y_{m}^{M}$

$Y_{j}^{J}$
Cost of purchasing tire type $p$ for collector $n$

Cost of purchasing recycled tires for all types of tires from recycler $r$

Holding cost of returned and produced tires per unit tire type $p$ at manufacturers

Holding cost of collected the scraped tires per unit tire type $p$ by collectors

Holding cost of produced tires for distributing per unit tire type $p$ at distributers

Maximum desired number of established sites for manufacturers

Maximum desired number of established sites for distributers

Maximum desired number of established sites for collectors

Normal distributed demand of market $l$ for tire type $p$

Mean value of demand of market $l$ for tire type $p$

Variance value of demand of market $l$ for tire type $p$

Normal distribution for the fraction of used tires type $p$ returned from market $l$.

Mean value of the fraction of used tires type $p$ returned from market $l$

Variance value of the fraction of used tires type $p$ returned from market $l$

Storage capacity of supplier $i$

Storage capacity of manufacturer $m$ for tire type $p$

Storage capacity of distributer $j$ for tire type $p$

Storage capacity of collector $n$ for tire type $p$

Storage capacity of recycler $r$ for tire type $p$

Safety stock of manufactured tire type $p$ at manufacturer $m$

Safety stock of collected tire type $p$ at collector $m$

Safety stock of supplier $i$

Safety stock of tire type $p$ at distributer $j$

Safety stock of recycled tire type $p$ at recycler $r$

Amount of products flow between supplier $i$ and manufacturer $m$ Amount of products flow between recycler $r$ and manufacturer $m$

Amount of tires type $p$ transformed between manufacturer $m$ and distributer $j$

Amount of tires type $p$ transformed between distributer $j$ and market $l$

Amount of returned tires type $p$ transformed between market $l$ and collector $n$

Amount of reused tires type $p$ transformed between collector $n$ and recycler $r$

1 if manufacturer $m$ is to be established, otherwise 0

1 if distributer $\mathrm{j}$ is to be established, otherwise 0 
$Y_{n}^{N} \quad 1$ if collector $\mathrm{n}$ is to be established, otherwise 0

\subsection{Mathematical formulation}

Regarding the proposed mathematical formulation, manufacturers (MNs) are the top-level, distributers (DCs) are the middle-level, and the collectors (COs) are the bottom-level in the framework of tire CLSC. Here, the proposed tri-level programming model is addressed as follows:

$$
\begin{aligned}
& M N: \min Z_{1}=\sum_{m=1}^{M} Y_{m}^{M} \times F C_{m}^{M}+\sum_{i=1}^{I} \sum_{m=1}^{M}\left(T C_{i m}^{I}+P C_{i}^{I}\right) \times X_{i m}^{I}+\sum_{r=1}^{R} \sum_{m=1}^{M}\left(T C_{r m}^{R}+P C_{r}^{R}\right) \times X_{r m}^{R} \\
& +\sum_{m=1}^{M} \sum_{p=1}^{P} \sum_{j=1}^{J}\left(M C_{m p}^{M}-P C_{m p}^{M}\right) \times X_{m j p}^{M}+\sum_{m=1}^{M} \sum_{p=1}^{P} H_{m p}^{M} \times \sum_{l=1}^{L} \frac{S_{m p}^{M}-d_{l p}^{\mu}}{d_{l p}^{\delta}} \times \sqrt{\sum_{j=1}^{J}\left(X_{m j p}^{M} \times d_{l p}^{\delta}\right)^{2}} \\
& \text { s.t. } \sum_{m=1}^{M} Y_{m}^{M} \leq M A X_{m} \\
& \sum_{r=1}^{R} X_{r m}^{R}+\sum_{i=1}^{I} X_{i m}^{I}=\sum_{j=1}^{J} \sum_{p=1}^{P} X_{m j p}^{M}, \forall m \\
& \sum_{m=1}^{M} X_{i m}^{I}+S_{i}^{I} \leq C A P_{i}^{I}, \forall i \\
& \sum_{m=1}^{M} X_{r m}^{R}+\sum_{p=1}^{P} S_{r p}^{R} \leq \sum_{p=1}^{P} C A P_{r p}^{R}, \forall r \\
& \sum_{j=1}^{J} X_{m j p}^{M}+S_{m p}^{M} \times Y_{m}^{M} \leq Y_{m}^{M} \times C A P_{m p}^{M}, \forall m, p \\
& Y_{m}^{M}=\{0,1\} \\
& \quad X_{i m}^{I}, X_{r m}^{R} \geq 0
\end{aligned}
$$

where $X_{m j p}^{M}$ is solved by the following level (second level):

$$
\begin{aligned}
& D C: \min Z_{2}=\sum_{j=1}^{J} Y_{j}^{J} \times F C_{j}^{J}+\sum_{m=1}^{M} \sum_{j=1}^{J} \sum_{p=1}^{P}\left(T C_{m j p}^{M}+P C_{m p}^{M}\right) \times X_{m j p}^{M}+\sum_{j=1}^{J} \sum_{l=1}^{L} \sum_{p=1}^{P}\left(T C_{j l p}^{J}-P C_{j p}^{J}\right) \times X_{j l p}^{J} \\
& +\sum_{j=1}^{J} \sum_{l=1}^{L} \sum_{p=1}^{P} M C_{j p}^{J} \times X_{j l p}^{J}+\sum_{j=1}^{J} \sum_{p=1}^{P} H_{j p}^{J} \times \sum_{l=1}^{L} \frac{S_{j p}^{J}-d_{l p}^{\mu}}{d_{l p}^{\delta}} \times \sqrt{\sum_{l=1}^{L}\left(X_{j l p}^{J} \times d_{l p}^{\delta}\right)^{2}} \\
& \text { S.t. } \sum_{j=1}^{J} Y_{j}^{J} \leq M A X_{j}
\end{aligned}
$$




$$
\begin{aligned}
& \sum_{m=1}^{M} X_{m j p}^{M}=\sum_{l=1}^{L} X_{j l p}^{J}, \forall j, p \\
& \sum_{j=1}^{J} X_{j l p}^{J} \leq d_{l p}, \forall l, p \\
& \sum_{l=1}^{L} X_{j l p}^{J}+Y_{j}^{J} \times S_{j p}^{J} \leq Y_{j}^{J} \times C A P_{j p}^{J}, \forall j, p \\
& \sum_{n=1}^{N} X_{\ln p}^{L} \leq a_{l p} \times d_{l p}, \forall l, p \\
& Y_{j}^{J}=\{0,1\} \\
& X_{m j p}^{M}, X_{l j p}^{L} \geq 0
\end{aligned}
$$

where $X_{\ln p}^{L}$ is solved by the following level (third level):

$$
\begin{aligned}
& C O: \min Z_{3}=\sum_{n=1}^{N} Y_{n}^{N} \times F C_{n}^{N}+\sum_{l=1}^{L} \sum_{n=1}^{N} \sum_{p=1}^{P}\left(T C_{\ln p}^{L}+P C_{l p}^{L}\right) \times X_{\ln p}^{L}+\sum_{n=1}^{N} \sum_{r=1}^{R} \sum_{p=1}^{P} T C_{n r p}^{N} \times X_{n r p}^{N} \\
& +\sum_{n=1}^{N} \sum_{p=1}^{P} \sum_{r=1}^{R}\left(M C_{n p}^{N}-P C_{n p}^{N}\right) \times X_{n r p}^{N}+\sum_{n=1}^{N} \sum_{p=1}^{P} H_{n p}^{N} \times \sum_{l=1}^{L} \frac{S_{n p}^{N}-\alpha_{l p}^{\mu} \times d_{l p}^{\mu}}{\alpha_{l p}^{\delta} \times d_{l p}^{\delta}} \times \sqrt{\sum_{r=1}^{R}\left(X_{n r p}^{N} \times \alpha_{l p}^{\delta} \times d_{l p}^{\delta}\right)^{2}} \\
& \text { s.t. } \sum_{n=1}^{N} Y_{n}^{N} \leq M A X_{n} \\
& \sum_{l=1}^{L} X_{\ln p}^{L}+Y_{n}^{N} \times S_{n p}^{N} \leq Y_{n}^{N} \times C A P_{n p}^{N}, \forall n, p \\
& \sum_{l=1}^{L} X_{\ln p}^{L}=\sum_{r=1}^{R} X_{n r p}^{N}, \forall n, p \\
& \sum_{n=1}^{N} X_{n r p}^{N}+S_{r p}^{R} \leq C A P_{r p}^{R}, \forall r, p \\
& Y_{n}^{N}=\{0,1\} \\
& \quad X_{\ln p}^{L}, X_{n r p}^{N} \geq 0
\end{aligned}
$$

As can be seen from the developed tri-level programming model, the equations (1) to (8) represent the first level for MNs' decisions. The equations (9) to (16) demonstrate the DCs' decision. Finally, the equations (17) to (23) indicate the decision level of COs. 
From the fist level, i.e. manufacturers (MNs), the objective function is represented in the equation (1). The first term determines the fixed opening cost regarding the locations of potential facilities for MNs. The second and third terms compute the transportation cost and purchasing cost for received raw materials from suppliers and or recyclers, respectively. Regarding the production costs, the manufacturing costs introducing the variable cost for manufacturing plants along with the profit of sold tires to distributers are considered, respectively. Finally, the holding cost or the storage cost is incurred due to inventory of manufactured and remanufactured tires. The last term is the sum of holding cost of manufacturers by ensuring that the safety stock can satisfy the corresponding demands.

The constraints of first level decision-making are presented by equations (2) to (8). Equation (2) shows the budget relayed to the maximum number of located facilities for the MNs. Equation (3) represents the flow of products between two different echelons. It means that the sum of raw materials from suppliers and recyclers should be equaled to the amount of manufactured tires distributed from MNs to DCs. The equations (4) to (6) show the maximum storage capacity of suppliers, recyclers, and MNs along with their safety stocks, respectively. The last two equations ensure the feasible amount of decision variables for the first level i.e. MNs.

The second level is to choose the DCs decisions. As such, the equation (9) is the objective function to minimize the total cost for DCs, and the equations (10) to (16) are the related constraints for the middle-level i.e. DCs. Regarding the objective function of this level, the first item aims to calculate the fixed opening cost corresponding to the location of DCs. The second term represents the transportation cost and purchasing cost for transformed tires between the manufactures to DCs. The third one calculates the transportation cost and the profits of sold tires to customers. Similarly, the fourth term shows the variable cost of distributing the tires to customers. Finally, the last term reveals the holding cost of distributers by controlling the safety stock which can satisfy the corresponding demands.

Regarding the constraints, the equation (10) illustrates the predefined maximum number of located DCs. The equation (11) reveals that all manufactured tires should be distributed among customers. Similarly, the equation (12) shows that all demand of customers should be met by DCs, as well. The equation (13) states that the flow of products through a DC is allowed only the DC is open and has enough storage capacity and safety stock as well. Furthermore, the equation (14) shows the amount of returned tires which should be collected by collectors (COs) is limited 
by a fraction of customer's demand. The last constraint of the second level refers the decision variables of DCs which are guaranteed by the equations (15) and (16).

The third level determined by COs maintains the reverse flows of used tires. The equation (17) considers the objective function of this level in five items. The first one shows the location decisions by calculating the fixed opening cost of facilities. The transportation cost and purchasing cost to collect the tires from markets and to transform them into recycling centers are considered by the second and third terms, respectively. The fourth item represents the variable cost for collecting of scraped tires along with the profits of COs from selling these collected tires to recyclers, as well. Eventually, the five item of objective function aims to calculate the holding cost of collectors by controlling the safety stock which can satisfy the corresponding demands of returned products.

From the constraints of COs level, the equation (18) by considering the fixed opening costs of COs, limits the number of established ones. Furthermore, the flow of collected scraped tires through a $\mathrm{CO}$ is allowed only that this facility must be opened and has enough storage capacity along with safety stock as can be seen from equation (19). Regarding the flow of returned products, the equation (20) shows that all collected tires should be recycled, as well. Equation (21) reveals the limitation of storage capacity and safety stock for recyclers. Finally, the equations (22) and (23) ensure the feasible amounts of decision variables of COs.

\section{Solution approach}

As mentioned earlier, the multi-level programming is NP-hard. Several approaches have been proposed to solve the bi-level and tri-level models. The solution methods for bi-level programming models can be divided into three classes [5]. The first category includes vertex enumeration methods [41]; [42]; [43]. The second classification is called Kuhn-Tucker algorithm [42]; [44]; [45]. In the last classification, penalty functions are used to tackle the bi-level programming models [46]. Hybridizing metaheuristics with explicit enumeration method has been done by Aksen and Nectai [47]. They utilized TS to solve the upper-level model and the solutions in the lower were validated by explicit enumeration method. Finally, an integrated approach by using metaheuristic was mentioned in the book written by Talbi [48]. The aim of this book was to introduce different metaheuristics including GA, PSO, TS and Differential Evolution (DE) [49] for solving the bi-level programming models. 
Some works that use tri-level models are: cutting plane algorithm [50], penalty function approach [51], Kuhn-Tucker transformation method [52], multi-parametric programming approach [53] and tri-level Kth-best algorithm [54]. The main drawback of such method is the poor performance when applying to high-dimensional case studies. To alleviate this limitation, heuristics techniques have been used in the literature. For instance, Mahmoodjanloo et al. [55] proposed two hybrid approaches based on the exact and metaheuristics. In their tri-level model, the first level was solved by a metaheuristic. The second level was evaluated by explicit enumeration algorithm and an exact approach was utilized for the third-level. In 2016, Han et al. [6] solved the tri-level programming model by PSO utilizing Kuhn-Tucker method for the lower levels as another method by hybridizing exact and metaheuristic solvers, simultaneously. Recently, Fathollahi Fard and Hajaghaei-Keshteli [19] introduced tri-level metaheuristic for solving the tri-level programming models for the first time.

In this work, we apply a simple nested approach called tri-level metaheuristic which uses metaheuristics for all levels to tackle the proposed tri-level programming model as well. We use SA and VNS as the popular metaheuristics existing in the literature along with KA and SFS as the two recent nature-inspired optimizers. The descriptions of these four algorithms are given in Supplementary Materials (F1). In addition, the main contribution of this study is to develop four new hybrid optimizers based on the benefits of recent and traditional metaheuristics. In the following, the structure of the proposed model and the process of solving it using a tri-level metaheuristic are discussed.

\subsection{Tri-level metaheuristic to solve the proposed tri-level mathematical model}

In multi-level programming, the variable of upper-level is the parameter in the lower-level. It means that all levels have a full knowledge about each other. The proposed multi-level model is a tri-level one. In this case, the first level is the leader and the two other levels are the followers. The proposed solution approach based on the developed tri-level mathematical model has been depicted by Figure 4. Accordingly, the considered model can be summarized as the following equations: 
As mentioned earlier, Talbi [48] proposed a nested approach to solve the bi-level model in which both levels are solved by metaheuristics. Fathollahi Fard and Hajaghaei-Keshteli [19] proposed a similar approach named tri-level metaheuristic. In this approach, the first level is first solved by metaheuristic approach using random solutions from lower levels. Then, the solution for this level is sent to the middle-level to solve the second one. These procedures are repeated for the third level. In the next iteration, the solution for lower level is sent back to upper level and the upper-level is resolved and this procedure will be continued [5]. Figure 4 shows the flowchart of the tri-level metaheuristic for the proposed tri-level model. It should be noted, according to Talbi [48], each level can be solved by only metaheuristic. In this paper, all levels are solved by the same metaheuristic to compare the methods.

\subsection{Encoding plan}

It is evident that, an encoding plan is needed to implement the employed algorithms when solving a discrete formulation by metaheuristics [5]. As such, a two-stage technique is utilized to set the problem for metaheuristics. Random-Key (RK) is a popular technique for representing a solution from a uniform distribution to a discrete one [56]. In RK, a solution is first created by random numbers. This solution can be used in a search space by various operators [57]. It is then converted to a feasible discrete problem by a procedure as illustrated here. This technique was utilized in several related papers to encode the mathematical models ([58][59][56][57] [2]).

In Figure 5, the proposed encoding method is shown. It can be seen that the solution is divided into two sub-solutions. Figure 5(a) shows the encoding plan for binary variables [3]. This subsolution chooses the location of potential facilities for each level. Figure 5(b) depicts a prioritybased representation to assign the facilities from different levels together. Regarding the literature, Gen et al. [60] proposed the priority-based representation in a two-stage transportation problem. This procedure is set by the sequence numbers and related on the capacity of facilities according to the constraints [56]. Figure 5(b) shows an example for allocation between the distributers and customers.

\subsection{Hybrid optimizers}


One of the main contributions of this paper is to utilize four new hybrid optimizers. The last decade has seen a rapid development in the area of nature-inspired metaheuristics resulting in several new algorithms including KA and SFS employed in this study [56][57][58]. The modifications and hybridization of recent metaheuristics have been popular methods too [58][59]. Such hybrid methods aggregate the advantages of different recent and old metaheuristic to improve the exploration and exploitation phases independently or combined [32]. In this work, four new hybrid optimizers based on the advantages of recent and traditional metaheuristics are proposed. In this regard, by using an intelligent design, each recent population-based metaheuristic as the main loop, is hybridized by a local search from the presented traditional single-solution ones. The following sub-sections are presented the four proposed algorithms.

\subsubsection{Hybrid of KA \& SA (HKASA)}

The KA as illustrated by Supplementary Materials F1.3 uses a strong exploitation phase by considering swirling and moving operators [58]. In the term of exploration phase, for each agent of N3 group, a set of population is generated randomly [2]. Although the randomization of procedure is good as the nature of metaheuristic, it can be modified by SA structures in per iteration as a sub-loop. Note that SA has been stated clearly in Supplementary Materials F1.1. Accordingly, each Keshtel is compared by the previous one and if it has been improved, it will be replaced. Conversely, SA structure for accepting the rejected solutions is considered by controlling a rate of temperature and its decreasing during the iterations. The proposed HKASA is addressed by the following pseudo-code as given in Figure 6.

\subsubsection{Hybrid of SFS \& VNS (HFVNS)}

In HFVNS, SFS explained by Supplementary Materials F1.4 plays the main actor and the rules of VNS considers as a local search to control the search phases in SFS. The diffusion operator in SFS is modified by a sub-loop from VNS which has been stated by Supplementary Materials F1.2. Instead of Gaussian distribution model, the procedures adopted from VNS are used. It seems that SFS is improved by exploration properties in the developed hybrid algorithm. The pseudo code of HFVNS is given in Figure 7.

\subsubsection{Hybrid of KA \& VNS (HKVNS)}

In the developed HKVNS, we want to keep the exploitation and exploration properties and reduce the time consumption in KA. Instead of three groups of Keshtels, only two of them will 
be used. N1 includes the set of lucky Keshtels who perform the attracting and swirling process. Also, N2 uses the rule of VNS by using the lucky ones. In the initial population, the best solutions are chosen by $N 1$ and the rest of them are $N 2$. In each iteration, $N 2 s$ are created by a neighbor from each agent of $N 1$ (i.e. lucky Keshtels). A VNS sub-loop is considered to do the exploration phase in this case. Figures 8 shows the steps of the HKVNS algorithm.

\subsubsection{Hybrid of SFS \& SA (HSFSA)}

In this hybrid algorithm, the diffusion operator is modified by employing the SA rules. In the term of exploitation phase, a local search is done as per the current temperature. This idea is probed to compare with its general algorithms in different criteria in the experiments. The pseudo-code of HSFSA is given in Figure 9. Generally speaking, the two hybrid algorithms presented for SFS increase the properties of exploration and exploitation phases for HFVNS and HSFSA, respectively. However, the two hybrid optimizers for KA improves the characteristics of exploration faze in two different ways i.e. HKASA and HKVNS.

\section{Experimental results}

In this section, the experimental setup is discussed including initial values for the parameters and generating benchmark problems. Taguchi experimental design method is then utilized to enhance the performance of algorithms. In order to check the metaheuristics, the results in small sizes are validated by an exact approach. In addition, the performances of employed optimizers are assessed by using different criteria. Finally, some sensitivity analyses are proposed to explore the behavior of decision makers in different cases when solving a real dataset. It should be noted that all experiments were done by a Core 2 Duo-2.26 GHz processor laptop. Also, the related codes were written in C++ and built in Microsoft Visual Studio 2014.

\subsection{Data generation}

To assess the optimizers, a set of benchmark problems with different levels of difficulties is needed [1]. Here, 24 test problems are designed with three difficulty levels (i.e. small, medium and large). Table 1 shows the test problems generated for the purpose of this work. 
The test problems are generated randomly as given the parameters in Table 2. In addition, the maximum desired number of facility in each decision level is estimated by a half of total number existing for this level.

It should be noted in order to approximate the value of fixed opening cost, storage capacity and safety stocks of facilities, the approach benchmarked from Devika et al. [1] has been utilized in our study.

\subsection{Taguchi experimental design method}

Since the algorithms employed and proposed in this work have several controlling parameter, the best value should be chosen to provide an unbiased comparison [2]. To do this, Taguchi method presented by Genichi Taguchi [61] is employed, in which, signal-to-noise $(S / N)$ ratio indicates the amount of variation in response variable. The higher value brings the better quality of this metric. $S / N$ ratio in the minimizing objective functions should be formulated as follows:

$$
S / N=-10 \log _{10}(\text { objective function })^{2}
$$

The best values for the parameters of the algorithms found by Taguchi method is shown in Table 3.

The test problems are solved 30 times and the following metric (Relative Percentage Deviation) is used to quantify their performance:

$$
R P D=\frac{\operatorname{Alg}_{\text {sol }}-\operatorname{Min}_{\text {sol }}}{\operatorname{Min}_{\text {sol }}}
$$

where $\operatorname{Min}_{\text {sol }}$ is the best solution among all solutions and $A l g_{\text {sol }}$ is the output of algorithm. The lower value of this metric brings the better quality. Moreover, Taguchi experimental design method proposes an orthogonal array to reduce the number of trials for each algorithm. According to Table 3, Taguchi approach offers $L_{9}$ for HFVNS. Also, $L_{16}$ is applied to SFS. For $\mathrm{SA}$ and VNS, $L_{18}$ is proposed. In addition, KA uses the orthogonal array of $L_{27}$. Finally, $L_{18}$ is considered by three other hybrid algorithms: HKASA, HKVNS and HSFSA. Due to page limitation, the results of $S / N$ ratio and mean $R P D$ for each algorithm are reported in Supplementary Materials F2. 
As a result, the tuned parameters for each presented metaheuristic are given by Table 4 .

\subsection{Validation of metaheuristics in small sizes}

This sub-section aims to check the quality of developed tri-level metaheuristics with an exact approach for tri-level programming model. A similar method to that in Fathollahi Fard and Hajaghaei-Keshteli [19] is used, in which the first and second levels are solved by an explicit enumeration method and the third level is tackled by an exact solver (i.e. GAMS in this study). This means that all levels were solved by exact method. In the proposed methodology, however, all levels are solved by metaheuristics. So, explicit enumeration method is used in this study to compare with metaheuristics. In the explicit enumeration algorithm (EX), all possible nodes should be considered. For instance, there are four potential manufacturers and two of them must be established. Therefore, $\left(\begin{array}{l}4 \\ 2\end{array}\right)$ nodes are existed and must be considered. In the small sizes, this approach is compared by different presented metaheuristics.

Table 5 shows the outputs of the methods. The Gap of best solutions is noticeable in the table. Figure 10(a) depicts the run time for all methods in this case by considering the exact method and tri-level metaheuristics. Besides, Figure 10(b) shows the metaheuristics behavior with each other. Hitting time is defined as the first time that an algorithm finds the best solution. The algorithms are compared by Figure 10(c).

Inspecting the results in Table 5, the quality of solutions for the metaheuristics is the same as the exact approach. The outputs of hybrid optimizers are better or same as recent metaheuristics. The standard deviation for VNS and SA are also more than the other algorithms. By contrast, HKASA shows a lower value. In term of hitting time VNS shows a better convergence rate for small sizes.

\subsection{Comparison of hybrid metaheuristics with their individual ones}

We run each algorithm 03 times and present the best, worst, average and standard deviation along with the run time and hitting time of employed optimizers in Table 6-7. For medium and 
large sizes, the behavior of algorithms in term of solution time and hitting time are depicted by Figure 11 and 12, respectively. As per the results in Figure 11, SA and VNS require less procedure time in all of test problems. Also, HKVNS shows less computational time as compared to hybrid and recent metaheuristics. Conversely, HKASA need a longer time to find a reasonable solution on the majority of test problems. Regarding the rate of hitting time for presented optimizers which can be seen from Figure 12, SA shows a good rate. However, SFS requires a more time to reach the best solution in the term of hitting time.

The behavior of algorithms in three sample problems (i.e. P10, P16 and P22) is compared in Figure 13. To have a fair comparison, the stop condition is the maximum number of iteration (200) for all algorithms. As can be seen, HKASA reaches shows the best convergence rate. The worst results are given by SA and VNS.

Finally, in order to verify the statistical validity of results, an analysis of variance (ANOVA) is done to confirm the significance of the results (based on RPD). This analysis is conducted for all test problems: small, medium and large sizes have been performed. The results demonstrate that there are substantial differences between the performances of algorithms. Figure 14 illustrates theses validities on the presented metaheuristics. In small-size problems, HKASA shows the best performance among all methods (See: Figure 14(a)). For medium-sizes problems, SFS provides the best performance as seen in Figure 14(b). In the large-size problems, it is evident that KA shows the worst results (See: Figure 14(c)). Meanwhile, there is a little difference between algorithms in large-size problem.

\subsection{Sensitivity analyses through a real data set}

In the last sub-section, a sensitivity analysis is conducted. A real case study is proposed to set the data of model. Our data set is generated from Mazandaran province in Iran. It is assumed that there are four suppliers, six manufacturers, ten distributers. In addition, 15 zones for demand markets are recognized. These zones are specified by geographical map as seen in Figure 15. Three collectors exist to collect the returned tires and send them to recyclers which are estimated 
as two centers. Besides, there are eighteen types of tires for different kinds of cars and trucks in our case study ${ }^{1}$.

The best algorithm found in the preceding section i.e. HKASA is used to perform the analysis. The behavior of all three decision levels in a one run is depicted by Figure 16. It is observed that regarding the nature of a tri-level programming model, in major of cases, there are some conflicting points between the decision levels of proposed problem.

As declared by results, the prices of forward and reverse system play a key role in the benefits of each level especially the leader i.e. manufacturers. By considering four different cases numbered as $\mathrm{C} 1$ to $\mathrm{C} 4$, the prices of recyclers are increased. The range of other parameters is similar to the estimated parameters in sub-section 4.1. Table 8 gives the outputs of the analysis. Figure 17 shows the effects of these changed on the objective function. According to the results, manufacturers choose the lower prices to provide the row materials. If the prices of recyclers are increased, the manufactures may be purchased the materials from supplier instead of recyclers.

\section{Conclusion and future works}

In this work, a tri-level decision-making model was proposed to address the tire closed-loop supply chain for the first time. Manufacturers, distributors and collectors were selected as the decision levels and the markets and recyclers and suppliers were the fixed levels. In order to address the developed model, an integrated approach utilizing metaheuristics for all levels, namely, tri-level metaheuristic was applied to tackle the problem. In addition, this paper developed four new hybrid optimizers based on the advantages of two recent metaheuristics i.e. KA and SFS and also two traditional local search-based algorithms i.e. SA and VNS. The validation of used methodology was checked by an exact algorithm. The algorithms were compared quantitatively and qualitatively to choose the best for solving a real case study. It was observed that HKASA was the best optimizer. Finally, a case study was employed to confirm the

\footnotetext{
${ }^{1}$ http://www.irantireco.com/en/employment/default.aspx
} 
merits of the proposed model. The results can be helpful by giving managerial insights for the tire industry.

Several future works can be recommended. The presented hybrid optimizers can be examined by other large-scale optimization problems. In addition, the results of methods can be compared by other heuristics and metaheuristics. The decision level for tire closed-loop supply chain can be changed by customers and recyclers. Also, the tri-level model may be extended to fourth-level to make the model more realistic. Moreover, some real constraints should be added for future works. For example, considering the vehicle routing operation for each center to develop the mathematical model would be a value continuation as well. 


\section{References}

[1] Devika, K., Jafarian, A., \& Nourbakhsh, V. (2014). Designing a sustainable closed-loop supply chain network based on triple bottom line approach: A comparison of metaheuristics hybridization techniques. European Journal of Operational Research, 235(3), 594-615.

[2] Fathollahi-Fard, A. M., \& Hajiaghaei-Keshteli, M., (2018). A Stochastic Multi-objective model for a Closedloop Supply Chain Problem with Environmental Considerations, Applied Soft Computing, 69, 232-249.

[3] Fathollahi Fard, A. M., Gholian-Jouybari, F., Paydar, M. M., \& Hajiaghaei-Keshteli, M. (2017). A bi-objective stochastic closed-loop supply chain network design problem considering downside risk. Industrial Engineering \& Management Systems, 16(3), 342-362.

[4] Farahani, R. Z., Rezapour, S., Drezner, T., \& Fallah, S. (2014). Competitive supply chain network design: An overview of classifications, models, solution techniques and applications. Omega, 45, 92-118.

[5] Fathollahi Fard, A. M., \& Hajiaghaei-Keshteli, M., (2018). A bi-objective partial interdiction problem considering different defensive systems with capacity expansion of facilities under imminent attacks. Applied Soft Computing, 68, 343-359.

[6] Han, J., Zhang, G., Hu, Y., \& Lu, J. (2016). A solution to bi/tri-level programming problems using particle swarm optimization. Information Sciences, 370, 519-537.

[7] Subulan, K., Taşan, A. S., \& Baykasoğlu, A. (2015). Designing an environmentally conscious tire closed-loop supply chain network with multiple recovery options using interactive fuzzy goal programming. Applied Mathematical Modelling, 39(9), 2661-2702.

[8] Collins, K. J., Jensen, A. C., Mallinson, J. J., Roenelle, V., \& Smith, I. P. (2002). Environmental impact assessment of a scrap tyre artificial reef. ICES Journal of Marine Science: Journal du Conseil, 59(suppl), S243-S249.

[9] Jang, J. W., Yoo, T. S., Oh, J. H., \& Iwasaki, I. (1998). Discarded tire recycling practices in the United States, Japan and Korea. Resources, conservation and recycling, 22(1), 1-14.

[10] Amin, S. H., Zhang, G., \& Akhtar, P. (2017). Effects of uncertainty on a tire closed-loop supply chain network. Expert Systems with Applications, 73, 82-91.

[11] Chopra, S., \& Meindl, P. (2015). Supply Chain Management: Strategy, Planning and Operation, 6th Edition, Pearson, New York.

[12] Govindan, K., Soleimani, H., \& Kannan, D. (2015). Reverse logistics and closed-loop supply chain: A comprehensive review to explore the future. European Journal of Operational Research, 240(3), 603-626.

[13] Govindan, K., \& Soleimani, H. (2017). A review of reverse logistics and closed-loop supply chains: a Journal of Cleaner Production focus. Journal of Cleaner Production, 142, 371-384.

[14] Cui, Y. Y., Guan, Z., Saif, U., Zhang, L., Zhang, F., \& Mirza, J. (2017). Close loop supply chain network problem with uncertainty in demand and returned products: Genetic artificial bee colony algorithm approach. Journal of cleaner production, 162, 717-742.

[15] Cheraghalipour, A., Paydar, M. M., \& Hajiaghaei-Keshteli, M., (2018). A bi-objective optimization for citrus closed-loop supply chain using Pareto-based algorithms. Applied Soft Computing, 69, 33-59.

[16] Ferrer, G. (1997). The economics of tire remanufacturing. Resources, conservation and recycling, 19(4), 221255.

[17] Kannan, G., Noorul Haq, A., \& Devika, M. (2009). Analysis of closed loop supply chain using genetic algorithm and particle swarm optimisation. International Journal of Production Research, 47(5), 11751200.

[18] Sasikumar, P., Kannan, G., \& Haq, A. N. (2010). A multi-echelon reverse logistics network design for product recovery - a case of truck tire remanufacturing. The International Journal of Advanced Manufacturing Technology, 49(9-12), 1223-1234.

[19] Fathollahi Fard, A. M., \& Hajaghaei-Keshteli, M. (2018). A tri-level location-allocation model for forward/reverse supply chain. Applied Soft Computing, 62, 328-346.

[20] Stackelberg, H. V. (1952). The Theory of the Market Economy. Oxford University Press, York. 
[21] Bracken, J., \& McGill, J. T. (1973). Mathematical programs with optimization problems in the constraints. Operations Research, 21(1), 37-44.

[22] Ben-Ayed, O. (1993). Bilevel linear programming. Computers \& operations research, 20(5), 485-501.

[23] Taniguchi, E., Noritake, M., Yamada, T., \& Izumitani, T. (1999). Optimal size and location planning of public logistics terminals. Transportation Research Part E: Logistics and Transportation Review, 35(3), 207-222.

[24] Huang, B., \& Liu, N. (2004). Bilevel programming approach to optimizing a logistic distribution network with balancing requirements. Transportation Research Record: Journal of the Transportation Research Board, (1894), 188-197.

[25] Kuo, R. J., \& Han, Y. S. (2011). A hybrid of genetic algorithm and particle swarm optimization for solving bilevel linear programming problem-A case study on supply chain model. Applied Mathematical Modelling, 35(8), 3905-3917.

[26] Ma, W., Wang, M., \& Zhu, X. (2014). Improved particle swarm optimization based approach for bilevel programming problem-an application on supply chain model. International Journal of Machine Learning and Cybernetics, 5(2), 281-292.

[27] Zhang, W., \& Xu, D. (2014). Integrating the logistics network design with order quantity determination under uncertain customer demands. Expert Systems with Applications, 41(1), 168-175.

[28] Saranwong, S., \& Likasiri, C. (2016). Product distribution via a bi-level programming approach: Algorithms and a case study in municipal waste system. Expert Systems with Applications, 44, 78-91.

[29] Saranwong, S., \& Likasiri, C. (2017). Bi-level programming model for solving distribution center problem: A case study in Northern Thailand's sugarcane management. Computers \& Industrial Engineering, 103, 2639.

[30] Parvasi, S. P., Mahmoodjanloo, M., \& Setak, M. (2017). A bi-level school bus routing problem with bus stops selection and possibility of demand outsourcing. Applied Soft Computing, 61, 222-238.

[31] Wolpert, D. H., \& Macready, W. G. (1997). No free lunch theorems for optimization. IEEE transactions on evolutionary computation, $1(1), 67-82$.

[32] Mirjalili, S., \& Gandomi, A. H., (2017). Chaotic gravitational constants for the gravitational search algorithm." Applied Soft Computing, 53, 407-419.

[33] Ma, X., Zhang, Q., Tian, G., Yang, J., \& Zhu, Z. (2018). On Tchebycheff Decomposition Approaches for Multiobjective Evolutionary Optimization. IEEE Transactions on Evolutionary Computation, 22(2), 226244.

[34] Tian, G., Zhou, M., \& Li, P. (2018a). Disassembly sequence planning considering fuzzy component quality and varying operational cost. IEEE Transactions on Automation Science and Engineering, 15(2), 748-760.

[35] Tian, Guangdong, Honghao Zhang, Yixiong Feng, Danqi Wang, Yong Peng, and Hongfei Jia. "Green decoration materials selection under interior environment characteristics: A grey-correlation based hybrid MCDM method." Renewable and Sustainable Energy Reviews 81 (2018b): 682-692.

[36] Mirjalili, S., Jangir, P., Mirjalili, S. Z., Saremi, S., \& Trivedi, I. N. (2017). Optimization of problems with multiple objectives using the multi-verse optimization algorithm. Knowledge-Based Systems, 134, 50-71.

[37] Hajiaghaei-Keshteli, M., \& Aminnayeri, M. (2013). Keshtel Algorithm (KA); a new optimization algorithm inspired by Keshtels' feeding. In Proceeding in IEEE Conference on Industrial Engineering and Management Systems (pp. 2249-2253).

[38] Salimi, H. (2015). Stochastic fractal search: a powerful metaheuristic algorithm. Knowledge-Based Systems, 75, $1-18$.

[39] Kirkpatrick, S., Gelatt, C. D., \& Vecchi, M. P. (1983). Optimization by simulated annealing. science, 220(4598), 671-680.

[40] Mladenović, N., \& Hansen, P. (1997). Variable neighborhood search. Computers \& Operations Research, 24(11), 1097-1100.

[41] Bard, J. F. (1998). Practical bilevel optimization: applications and algorithms.

[42] Shi, C., Lu, J., \& Zhang, G. (2005). An extended Kth-best approach for linear bilevel programming. Applied Mathematics and computation, 164(3), 843-855. 
[43] Zhang, G., Shi, C., \& Lu, J. (2008). An Extended K th-Best Approach For Referential-Uncooperative Bilevel Multi-Follower Decision Making. International Journal of Computational Intelligence Systems, 1(3), 205214.

[44] Bard, J. F., \& Falk, J. E. (1982). An explicit solution to the multi-level programming problem. Computers \& Operations Research, 9(1), 77-100.

[45] Hansen, P., Jaumard, B., \& Savard, G. (1992). New branch-and-bound rules for linear bilevel programming. SIAM Journal on scientific and Statistical Computing, 13(5), 1194-1217.

[46] White, D. J., \& Anandalingam, G. (1993). A penalty function approach for solving bi-level linear programs. Journal of Global Optimization, 3(4), 397-419.

[47] Aksen, D., \& Aras, N. (2012). A bilevel fixed charge location model for facilities under imminent attack. Computers \& Operations Research, 39(7), 1364-1381.

[48] Talbi, E. G. (2013). Metaheuristics for bi-level optimization (Vol. 482). Springer.

[49] Storn, R., \& Price, K. (1997). Differential evolution-a simple and efficient heuristic for global optimization over continuous spaces. Journal of global optimization, 11(4), 341-359.

[50] Bard, J. F. (1984). An investigation of the linear three level programming problem. IEEE Transactions on Systems, Man, and Cybernetics, (5), 711-717.

[51] White, D. J. (1997). Penalty function approach to linear trilevel programming. Journal of Optimization Theory and Applications, 93(1), 183-197.

[52] Anandalingam, G. (1988). A mathematical programming model of decentralized multi-level systems. Journal of the Operational Research Society, 39(11), 1021-1033.

[53] Faísca, N. P., Saraiva, P. M., Rustem, B., \& Pistikopoulos, E. N. (2009). A multi-parametric programming approach for multilevel hierarchical and decentralised optimisation problems. Computational management science, 6(4), 377-397.

[54] Zhang, G., Lu, J., Montero, J., \& Zeng, Y. (2010). Model, solution concept, and Kth-best algorithm for linear trilevel programming. Information Sciences, 180(4), 481-492.

[55] Mahmoodjanloo, M., Parvasi, S. P., \& Ramezanian, R. (2016). A tri-level covering fortification model for facility protection against disturbance in r-interdiction median problem. Computers \& Industrial Engineering, 102, 219-232.

[56] Sadeghi-Moghaddam, S., Hajiaghaei-Keshteli, M., \& Mahmoodjanloo, M. (2017). New approaches in metaheuristics to solve the fixed charge transportation problem in a fuzzy environment. Neural Computing and Applications, 1-21.

[57] Samadi, A., Mehranfar, N., Fathollahi Fard, A. M., \& Hajiaghaei-Keshteli, M. (2018). Heuristic-based metaheuristics to address a sustainable supply chain network design problem. Journal of Industrial and Production Engineering, 35 (2), 102-117.

[58] Hajiaghaei-Keshteli, M., \& Fathollahi Fard, A. M. (2018). Sustainable closed-loop supply chain network design with discount supposition. Neural Computing and Applications, 1-35.

[59] Golmohamadi, S., Tavakkoli-Moghaddam, R., \& Hajiaghaei-Keshteli, M. (2017). Solving a fuzzy fixed charge solid transportation problem using batch transferring by new approaches in meta-heuristic. Electronic Notes in Discrete Mathematics, 58, 143-150.

[60] Gen, M., Altiparmak, F., \& Lin, L., (2006). A genetic algorithm for the two-stage transportation problem using priority-based encoding, OR Spectrum, 28, 337-354.

[61] Taguchi, G. (1986). Introduction to quality engineering: designing quality into products and processes. 


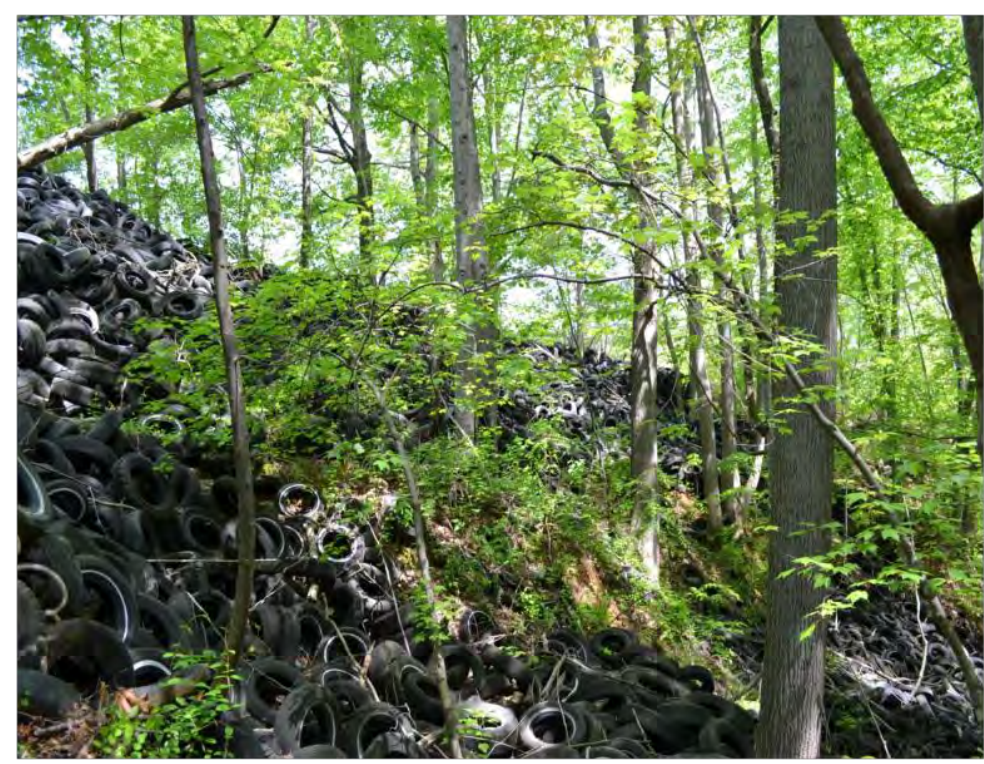

Figure 1. The scraped tires may be left in nature to pollute the environment (this photo is taken from our case study)

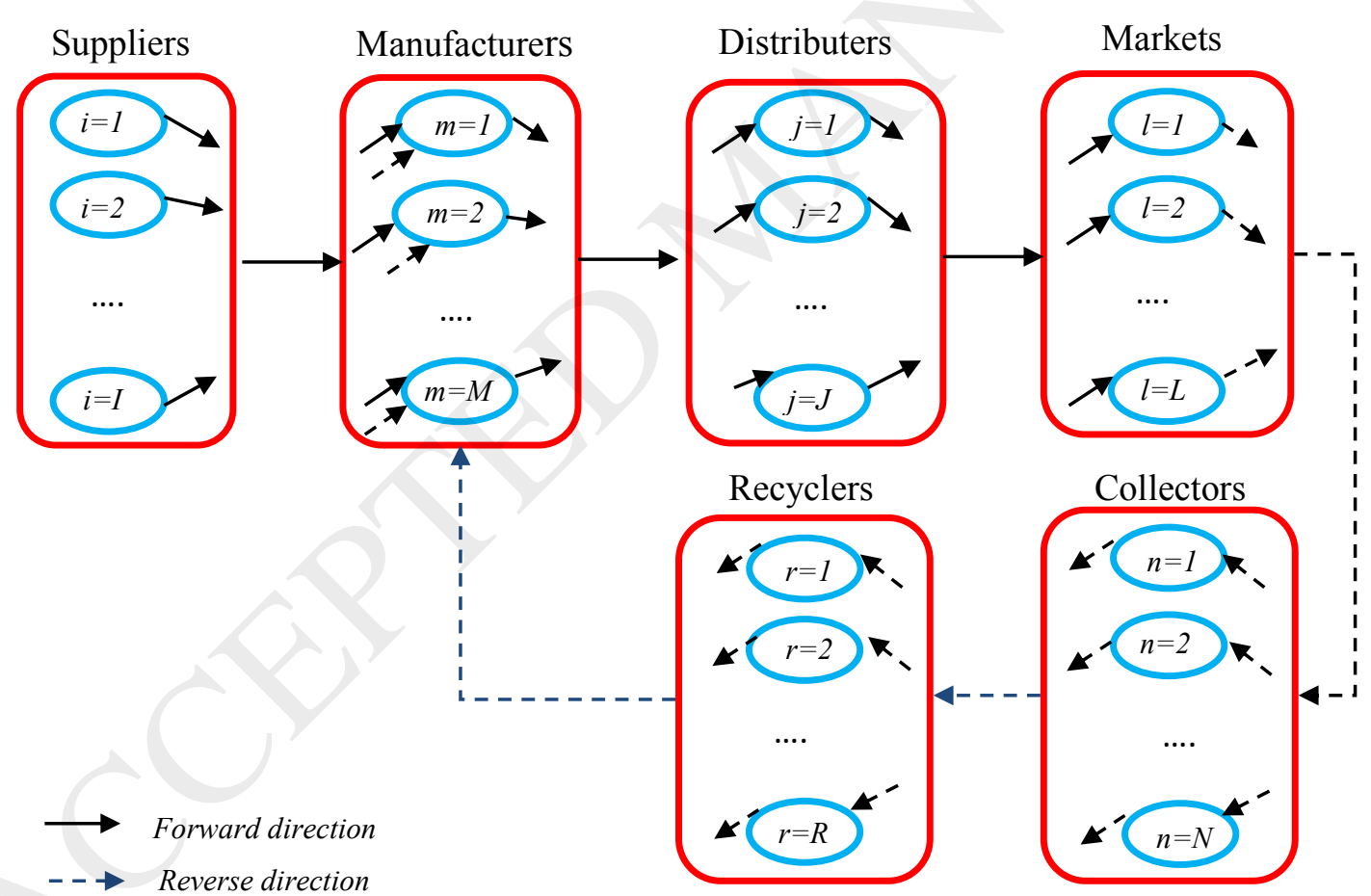

Figure 2. The graphical structure of tire closed-loop supply chain 


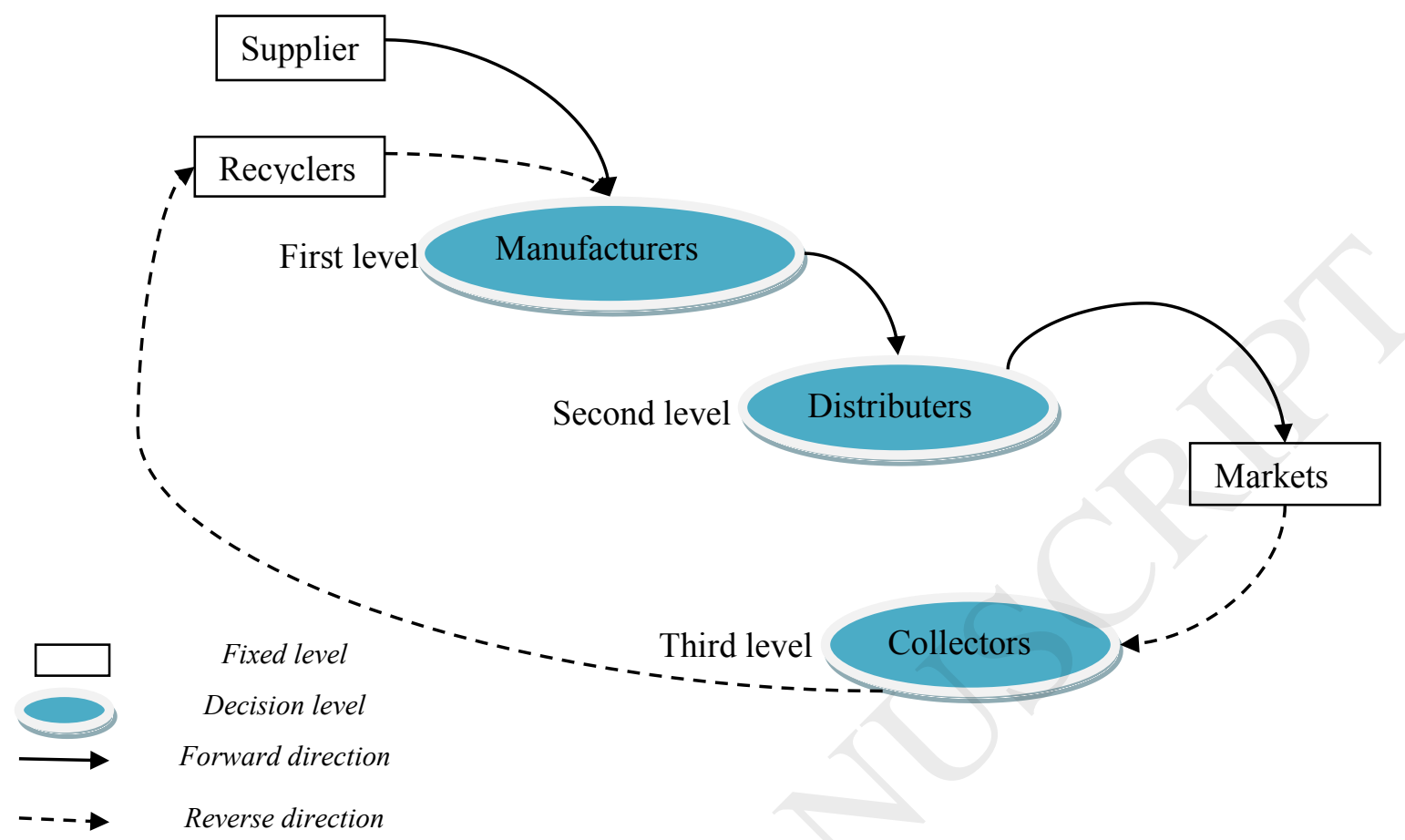

Figure 3. The structure of tri-level programming model for the closed-loop supply chain 


\section{Start}

\section{Initialization}

Set of parameters

Initialize a population of solutions or a single solution

$$
X\left(Y_{m}^{M}, Y_{j}^{J}, Y_{n}^{N}, X_{r m}^{R} \mid X_{i m}^{I}, X_{m j p}^{M}, X_{j l p}^{J}, X_{\ln p}^{L}, X_{n r p}^{N}\right)
$$

First level solving

Metaheuristic to solve the leader

Decision variable of $X\left(Y_{m}^{M}, X_{r m}^{R}, X_{i m}^{I}\right)$ is solved

\section{Second level solving}

Metaheuristic to solve the middlelevel follower

Decision variable of $X\left(Y_{m}^{M}, Y_{j}^{J}, X_{r m p}^{R}, X_{i m}^{I}, X_{m j p}^{M}, X_{j l p}^{J}\right)$ is solved

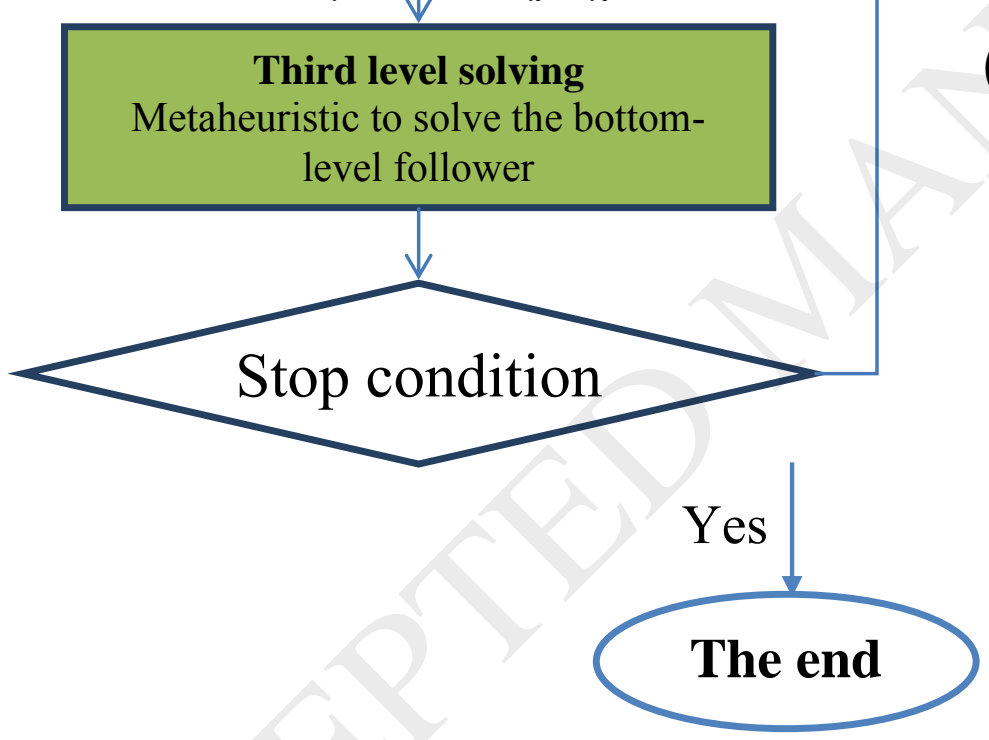

$\left(Z_{1}\right)$

$\left(Z_{2}\right)$

$\left(Z_{3}\right)$

\section{$\mathrm{NO}$}

Figure 4. Constructing of the tri-level metaheuristic to solve the proposed tri-level programming model [19] 


\begin{tabular}{|l|l|c|c|c|}
$P_{1}$ & $P_{2}$ & $P_{3}$ & $P_{4}$ & $P_{5}$ \\
\hline 0.34 & 0.57 & 0.25 & 0.68 & 0.92 \\
\hline$M A X$ & $=3$ \\
\hline & \multicolumn{5}{|l}{} \\
\hline 0 & 1 & 0 & 1 & 1 \\
\hline
\end{tabular}

(a) The graphical illustration of encoding plan for the binary variables ( $Y_{\square}^{\square}$ )

RK:

Sequence:

Order:

\begin{tabular}{|l|l|l|l|l|l|l|}
\hline 0.44 & 0.41 & 0.56 & 0.94 & 0.12 & 0.73 & 0.42 \\
\hline 2 & 1 & 3 & 4 & 1 & 3 & 2 \\
\hline 1 & 2 & 3 & 1 & 2 & 3 & 4 \\
\hline Distributers
\end{tabular}

The steps of allocation re as follows:

\begin{tabular}{|l|l|l|l|l|}
\hline 2 & 3 & 4 & 3 & 2 \\
\hline 1 & 3 & 1 & 3 & 4 \\
\hline
\end{tabular}

The second customer

rchases from the second distributer

\begin{tabular}{|l|l|l|}
\hline 3 & 4 & 3 \\
\hline 3 & 1 & 3 \\
\hline
\end{tabular}

The fourth customer chases from the first distributer

The first and third customers purchase from third distributer

(b). An example for the priority-based representation: flow of products and allocation

Figure 5.The proposed encoding plan in two sub-solutions (i.e. (a) and (b)) 
Initialize Keshtels population

Calculate the fitness and sort them in three types: $N_{1}, N_{2}$ and $N_{3}$

$\mathrm{X}^{*}=$ the best solution.

while ( $t<$ maximum number of iteration)

for each $N_{l}$

Calculate the distance between this lucky Keshtel and all Keshtels.

Select the closest neighbor.

$\mathrm{S}=0$;

while $(\mathrm{S}<$ maximum number of swirling)

Do the swirling.

if the fitness of this new position is better than prior

Update this lucky Keshtel.

break

endif

$\mathrm{S}=\mathrm{S}+1$

endwhile

endfor

for each $N_{2}$

Move the Keshtel between the two Keshtels.

\section{endfor}

for each $N_{3}$

Generate a new Keshtel as a new random solution.

if the function value of the new solution is better than prior

Replace the new solution as old solution.

else

Calculate $\delta, \delta=\left|f_{\text {old }}-f_{\text {new }}\right|$.

if rand $<\exp (-\delta / T)$

Replace the new solution.

endif

endif

endfor

Update T;

Merge the $N_{l}, N_{2}$ and $N_{3}$

Sort the Keshtels and form $N_{1}, N_{2}$ and $N_{3}$ for next iteration.

Update the $\mathrm{X}^{*}$ if there is better solution.

$\mathrm{t}=\mathrm{t}+1$;

end while

return $X^{*}$

Figure 6. The pseudo-code of HKASA 
Set the parameters and initialize a population of $N$ points

A set of neighborhood structures $N_{k}$ for $k=1,2, \ldots, k_{\max }$ for shaking.

$\mathrm{BP}=$ the best point.

while ( $t<$ maximum number of iteration)

for each point $P_{i}$

/*Do the diffusion process*/

$\mathrm{k}=1$;

while $\left(k<k_{\max }\right)$

Shaking: pick a random solution $P_{i}$ from the $k^{\text {th }}$ neighborhood $N_{k}\left(P_{i}\right)$ of $P_{i}$

$P_{i}{ }^{\prime}=$ local search $\left(P_{i}\right)$;

if the objectives function of $P_{i}{ }^{\prime}$ is better than $P_{i}$

$P_{i}=P_{i}^{\prime}$;

Continue to search with $N_{l} ; \mathrm{k}=1$;

else

$\mathrm{k}=\mathrm{k}+1$;

endif

endwhile

endfor

Do the updating process.

*/the first updating process/*

All points are ranked by the following equation:

$P a_{i}=\frac{\operatorname{rank}\left(P_{i}\right)}{N}$

for each point $P_{i}$

if $\mathrm{rand}>P a_{i}$

Updating the position based on the following equation:

$P_{i}^{\prime}=P_{i}-\varepsilon^{\prime} \times\left(P_{t}^{\prime}-P_{i}\right)$

endif

endfor

*/the second updating process/*

All points are ranked by the following equation:

$$
P a_{i}=\frac{\operatorname{rank}\left(P_{i}\right)}{N}
$$

for each point $P_{i}$

if rand $>P a_{i}$

Updating the position based on the following equations:

$P_{i}^{\prime \prime}=P_{i}^{\prime}-\varepsilon^{\prime} \times\left(P_{t}^{\prime}-B P\right), \mid \varepsilon^{\prime} \leq 0.5$

$P_{i}^{\prime \prime}=P_{i}^{\prime}-\varepsilon^{\prime} \times\left(P_{t}^{\prime}-P_{r}^{\prime}\right), \mid \varepsilon^{\prime}>0.5$

\section{endif}

endfor

Update the PB if there is better point.

$\mathrm{t}=\mathrm{t}+1$;

end while

return $\mathrm{BP}$

Figure 7. The pseudo-code of HFVNS 
Initialize Keshtels population

Calculate the fitness and sort them in three types: $N_{l}$ and $N_{2}$.

A set of neighborhood structures $N_{k}$ for $k=1,2, \ldots, k_{\text {max }}$ for shaking.

$\mathrm{X}^{*}=$ the best solution.

while $(t<$ maximum number of iteration)

for each $N_{l}(L K)$

Calculate the distance between this lucky Keshtel and all Keshtels.

Select the closest neighbor.

$\mathrm{S}=0$;

while $(\mathrm{S}<$ maximum number of swirling)

Do the swirling.

if the fitness of this new position is better than prior

Update this lucky Keshtel.

break

endif

$\mathrm{S}=\mathrm{S}+1$

endwhile

endfor

for each $N_{2}$

$\mathrm{k}=1$;

while $\left(k<k_{\max }\right)$

Shaking: pick a random solution $N_{2}$ from the $k^{\text {th }}$ neighborhood $N_{k}(L K)$ of $N_{2}$.

$N_{2}{ }^{\prime}=$ local search $(L K)$;

if the objectives function of $N_{2}$ ' is better than $N_{2}$

$P_{i}=P_{i}$;

Continue to search with $N_{l} ; \mathrm{k}=1$;

else

$\mathrm{k}=\mathrm{k}+1$;

endif

endwhile

endfor

Merge the $N_{l}$ and $N_{2}$

Sort the Keshtels and form $N_{1}$ and $N_{2}$ for next iteration.

Update the $\mathrm{X}^{*}$ if there is better solution.

$\mathrm{t}=\mathrm{t}+1$;

end while

return $X^{*}$

Figure 8. The pseudo-code of HKVNS 


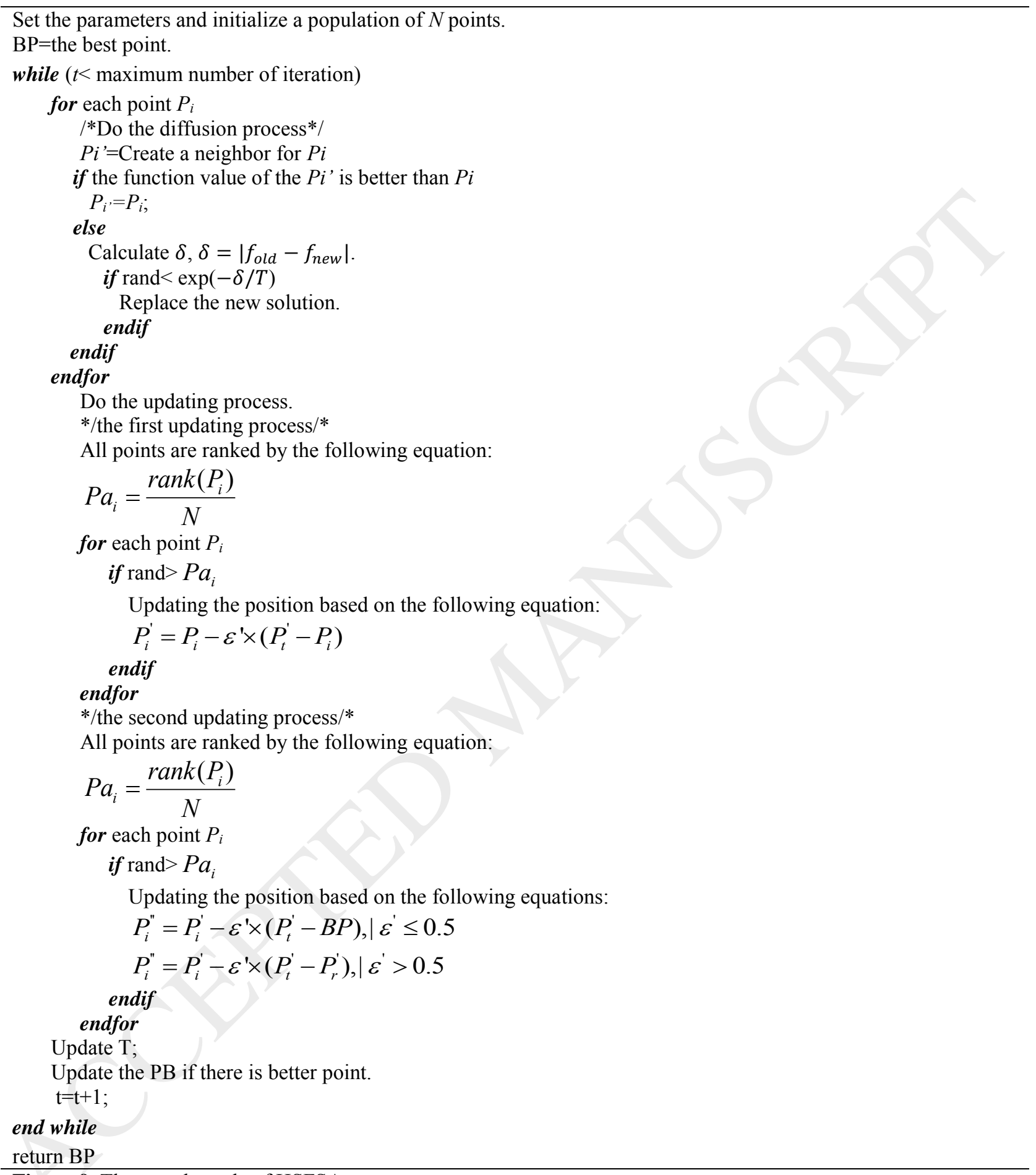

Figure 9. The pseudo-code of HSFSA 


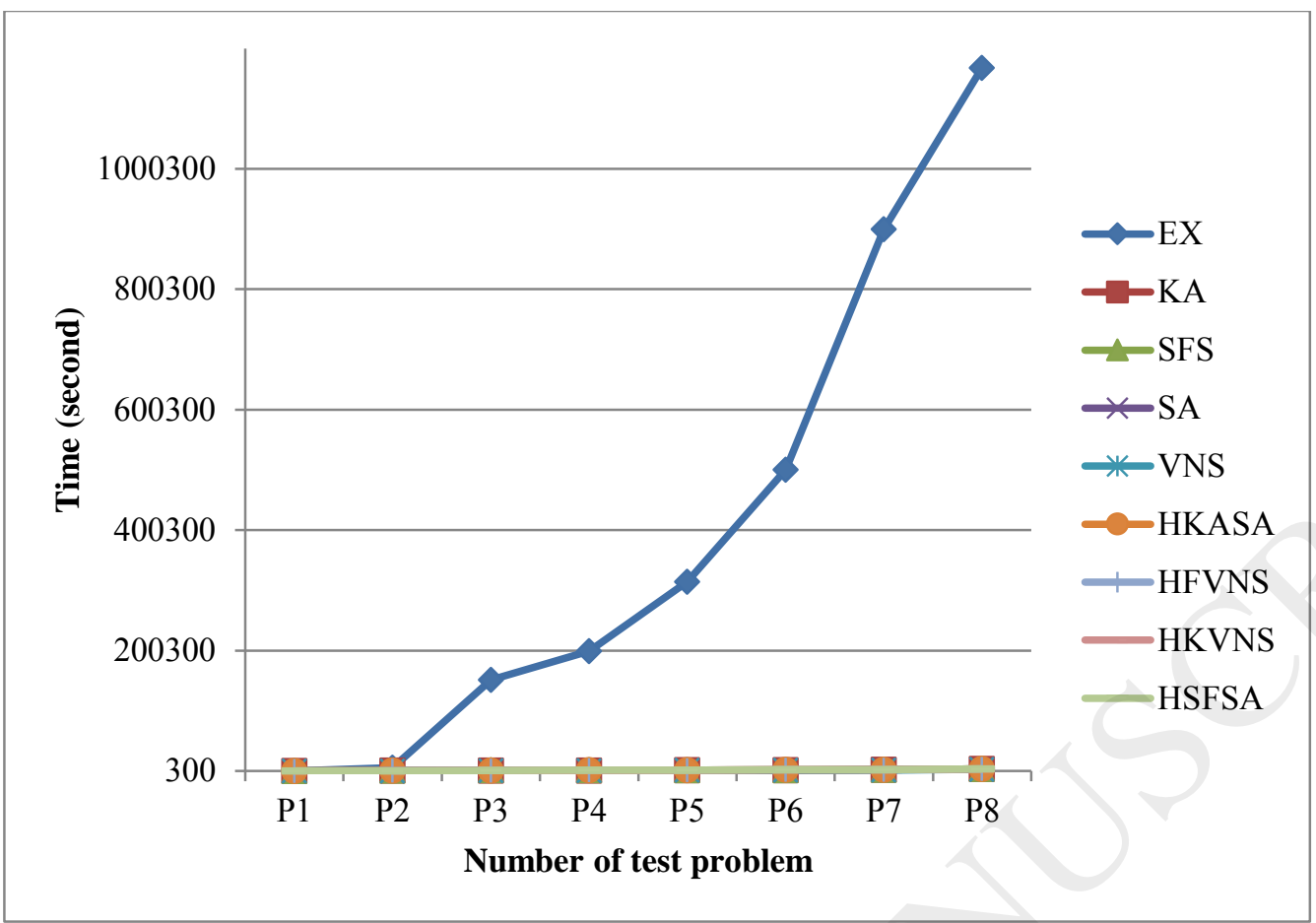

(a)

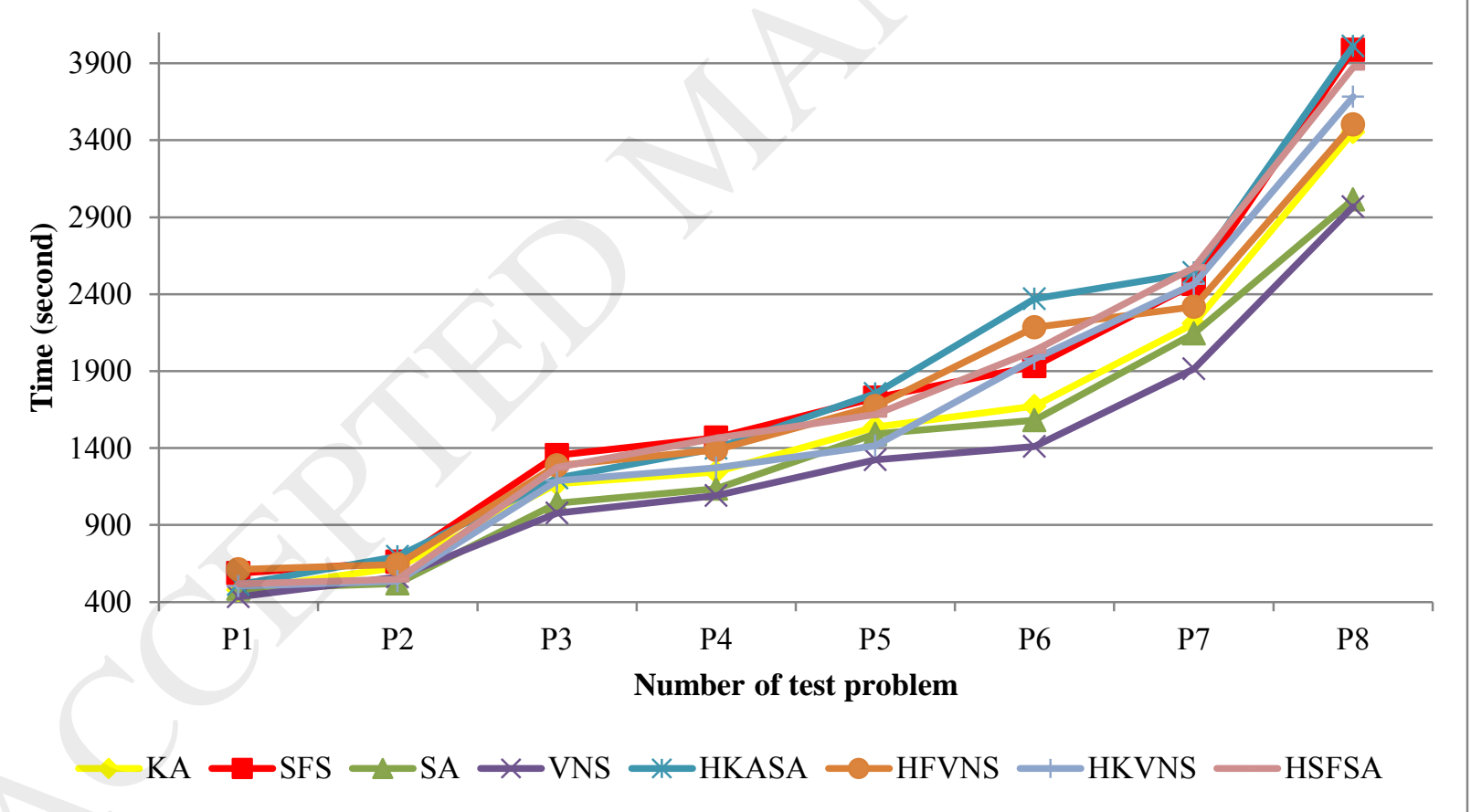

(b) 


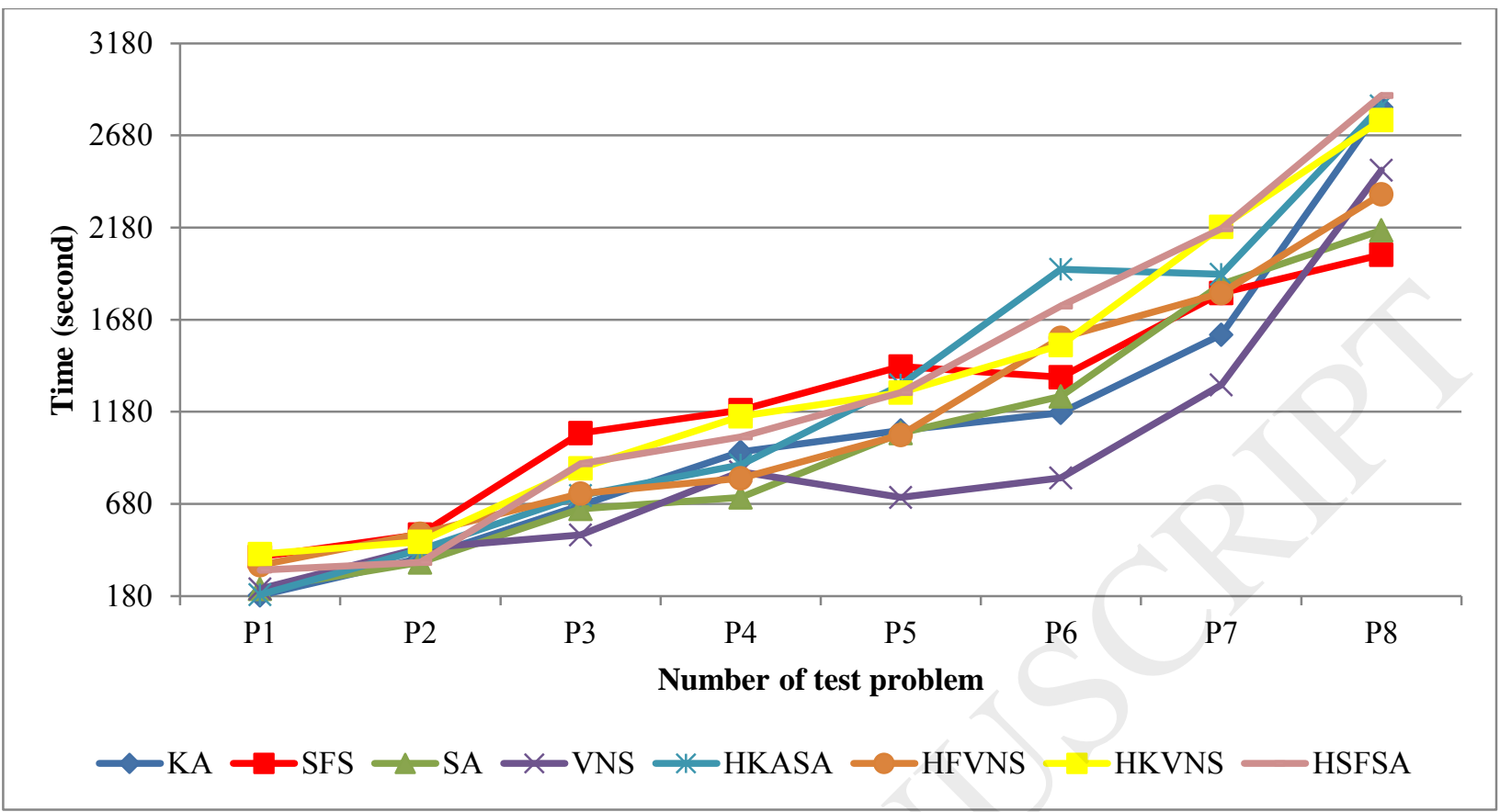

(c)

Figure 10. The behavior of methods in term of CPU and HT for small sizes (i.e. (a) for CPU by considering the exact method, (b) CPU without the exact method and (c) for HT for metaheuristics) 


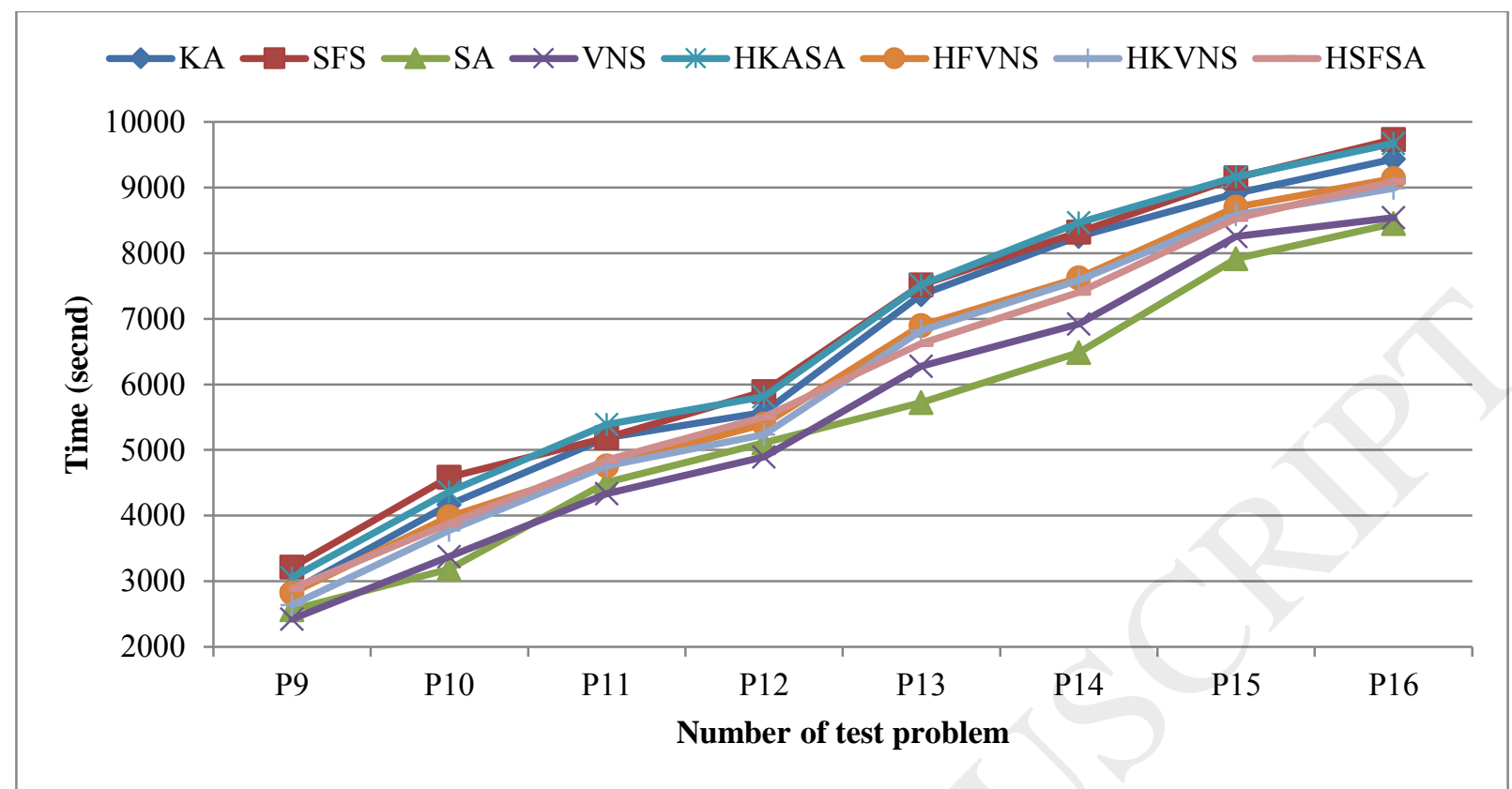

(a)

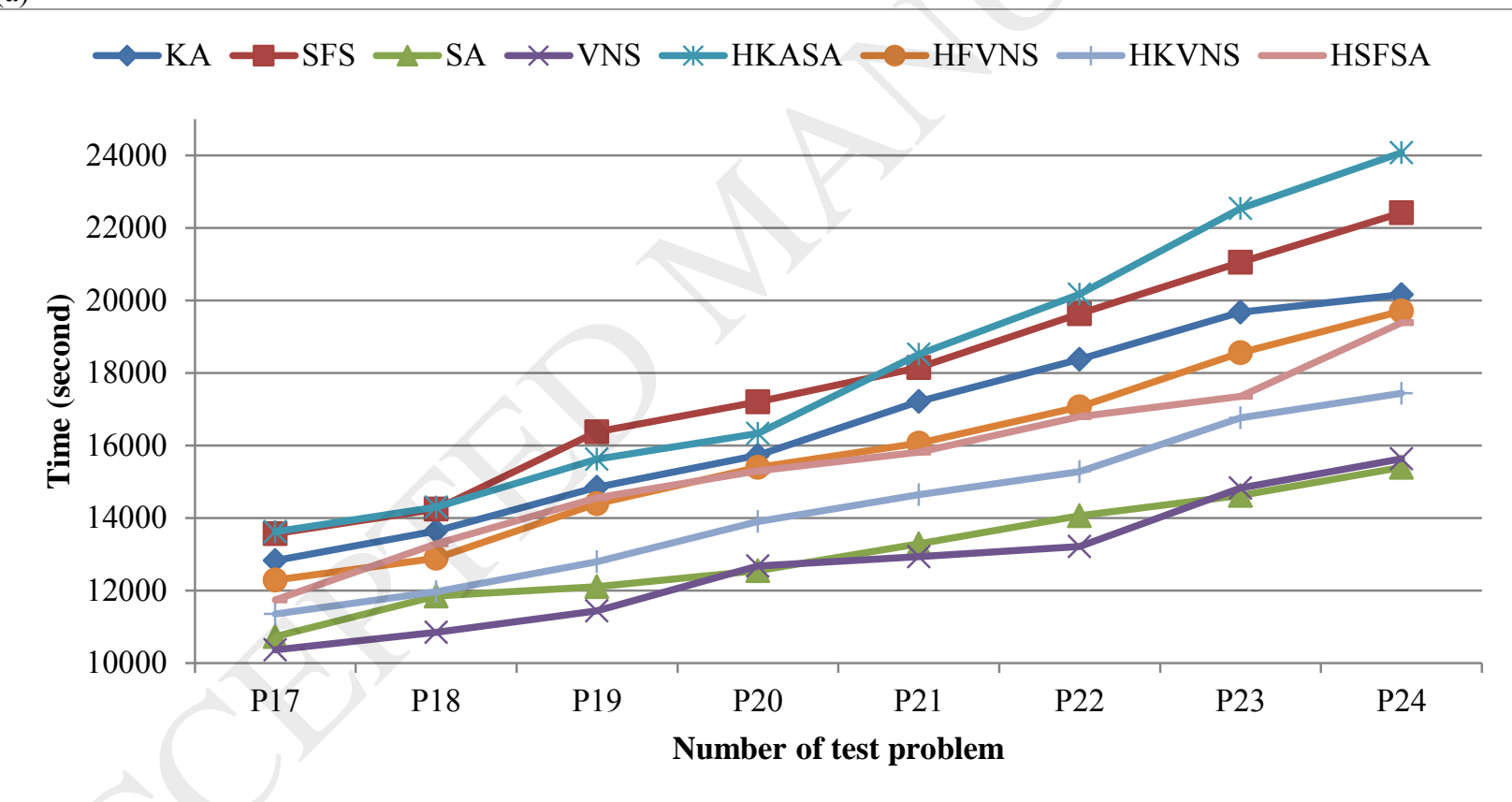

(b)

Figure 11. The behavior of methods in term of solution time (i.e. (a) for medium sizes and (b) for large sizes) 


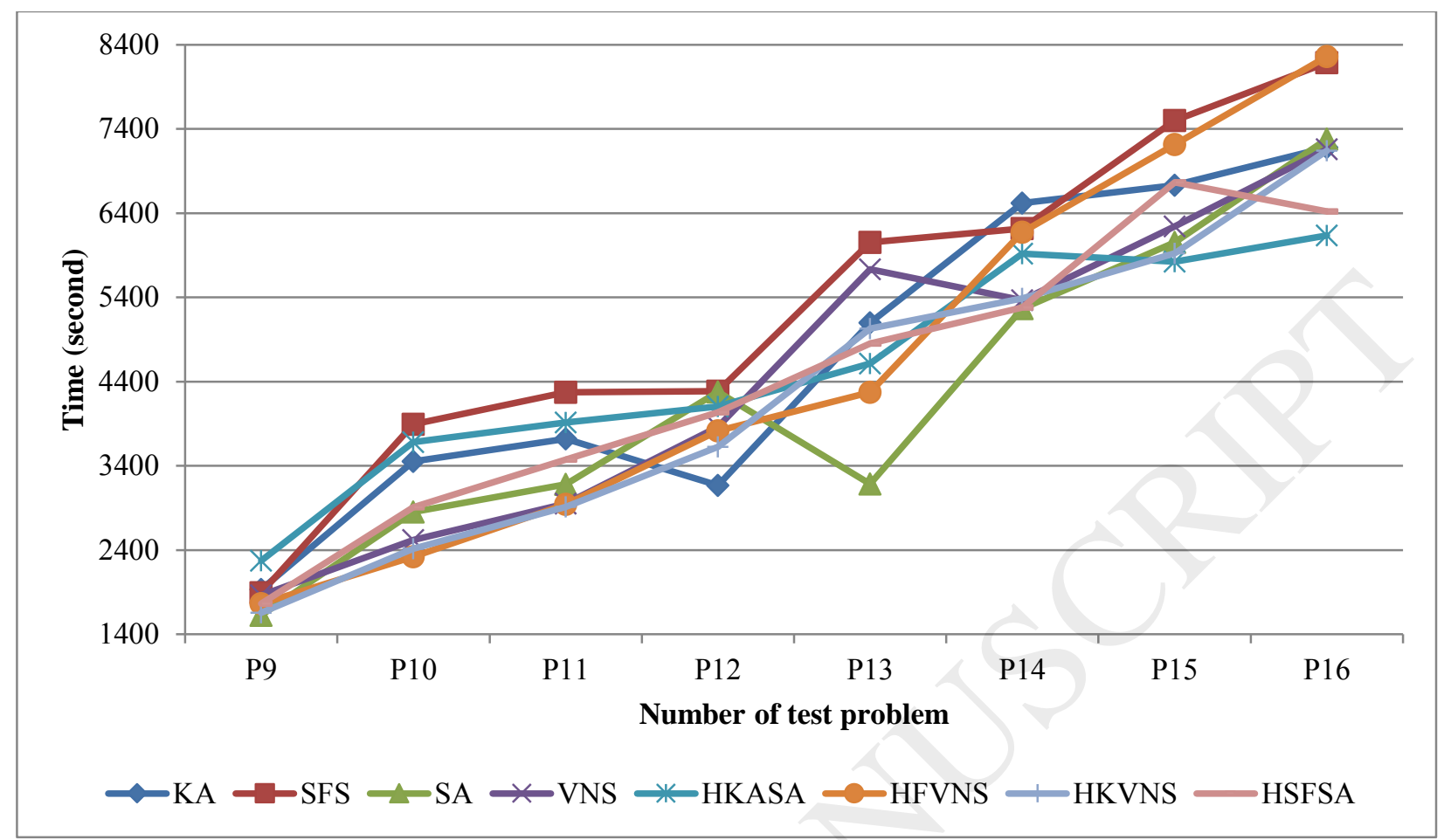

(a)

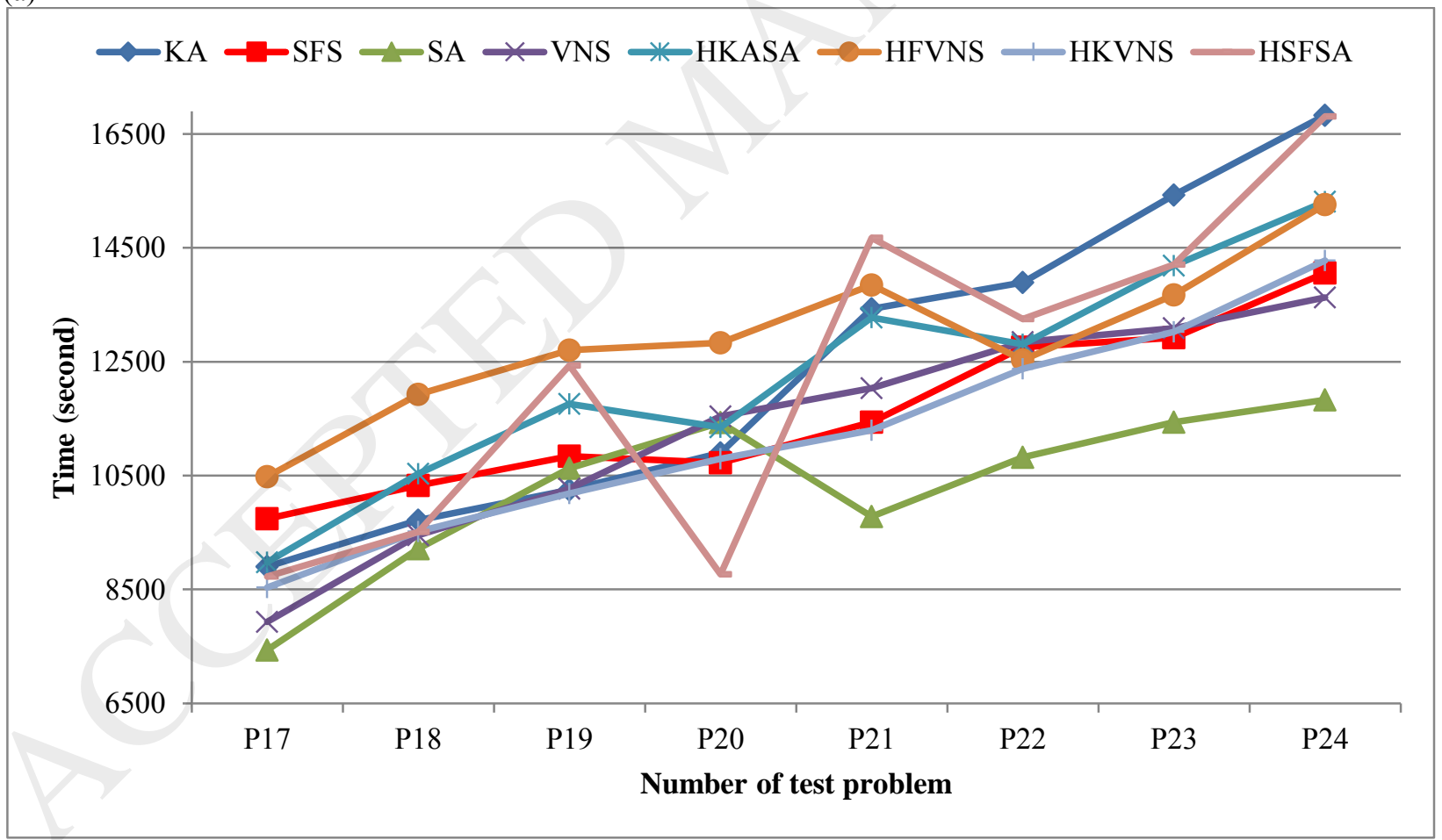

(b)

Figure 12. The behavior of methods in term of hitting time (i.e. (a) for medium sizes and (b) for large sizes) 


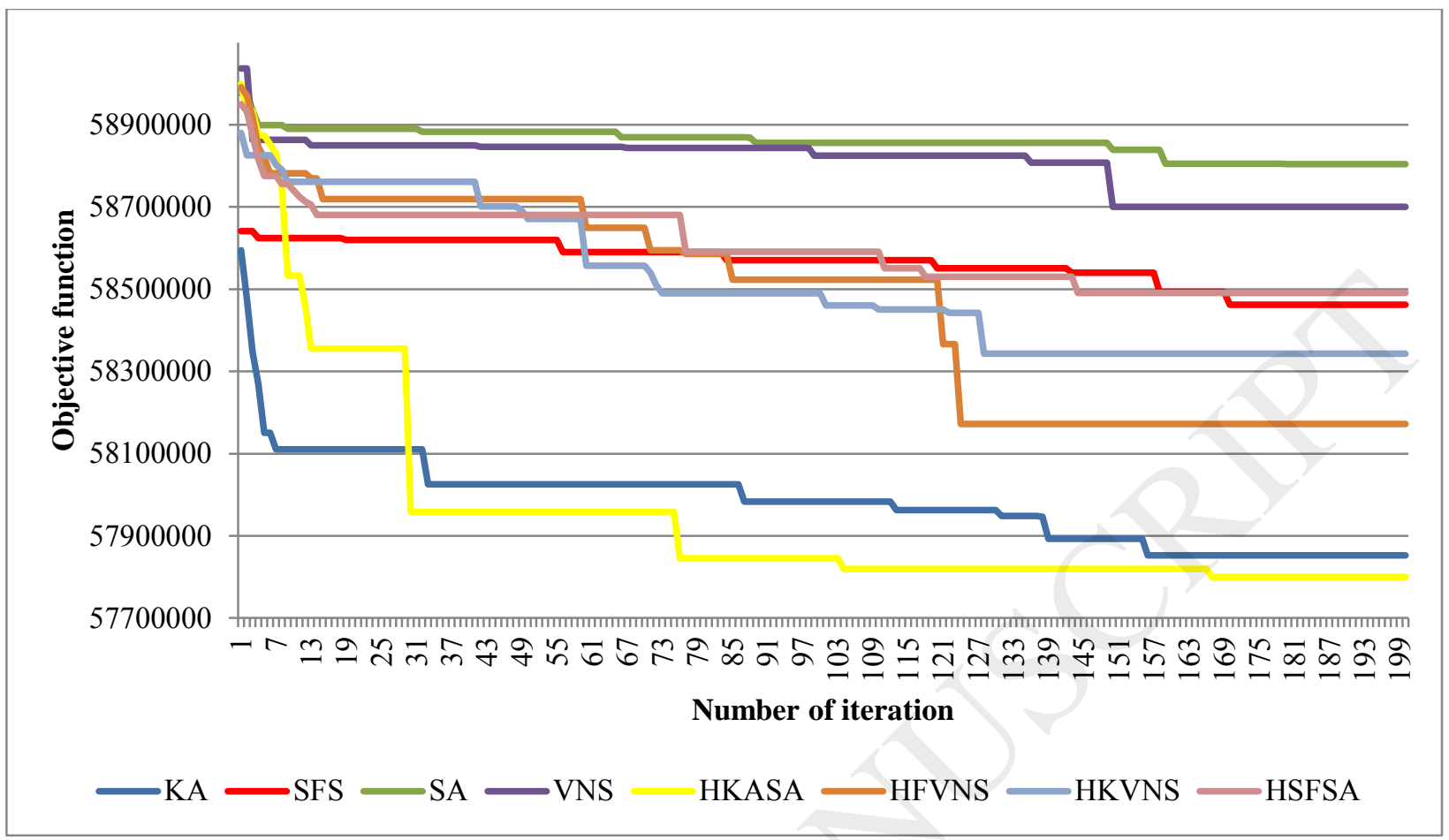

(a)

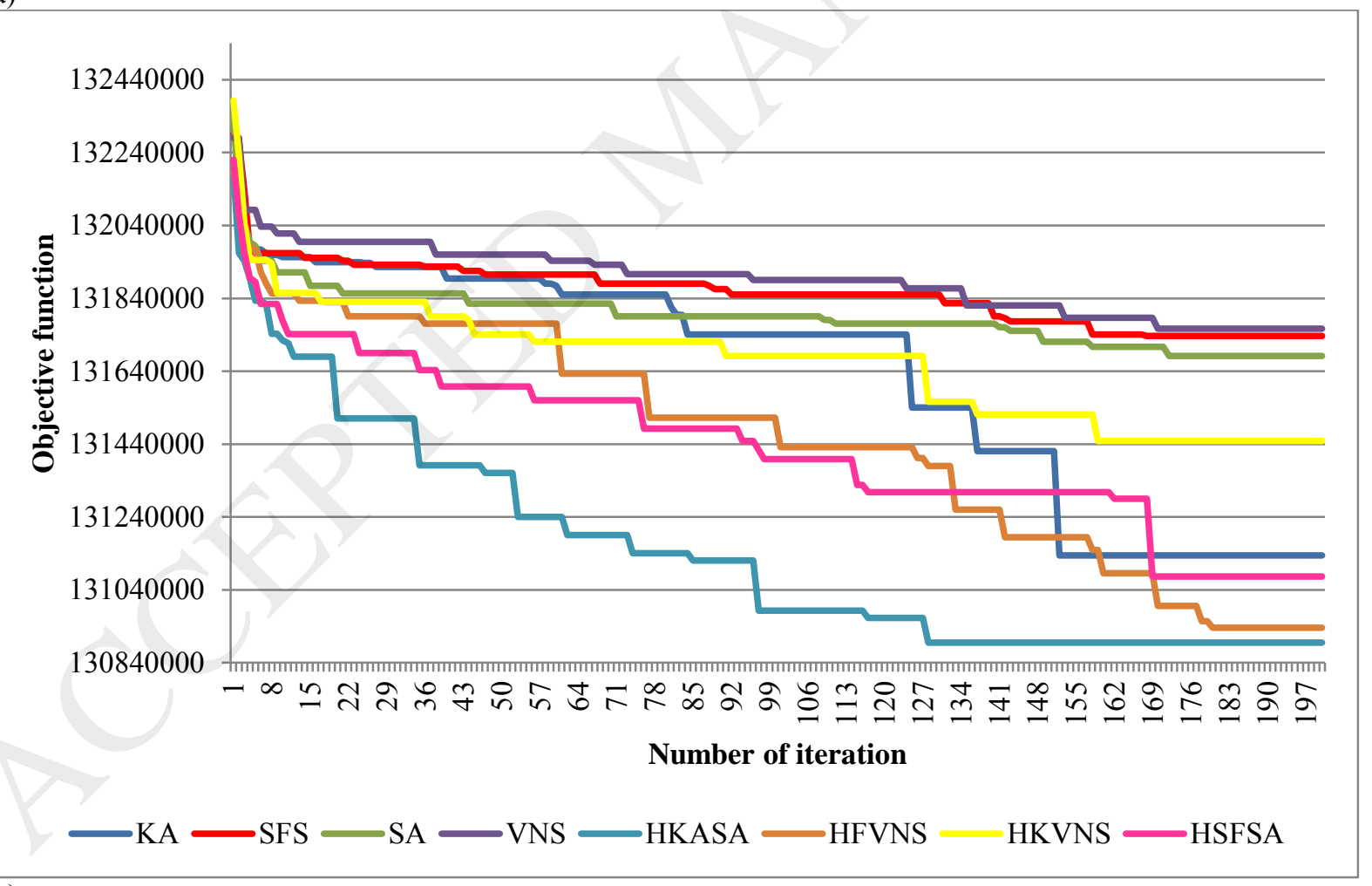

(b) 


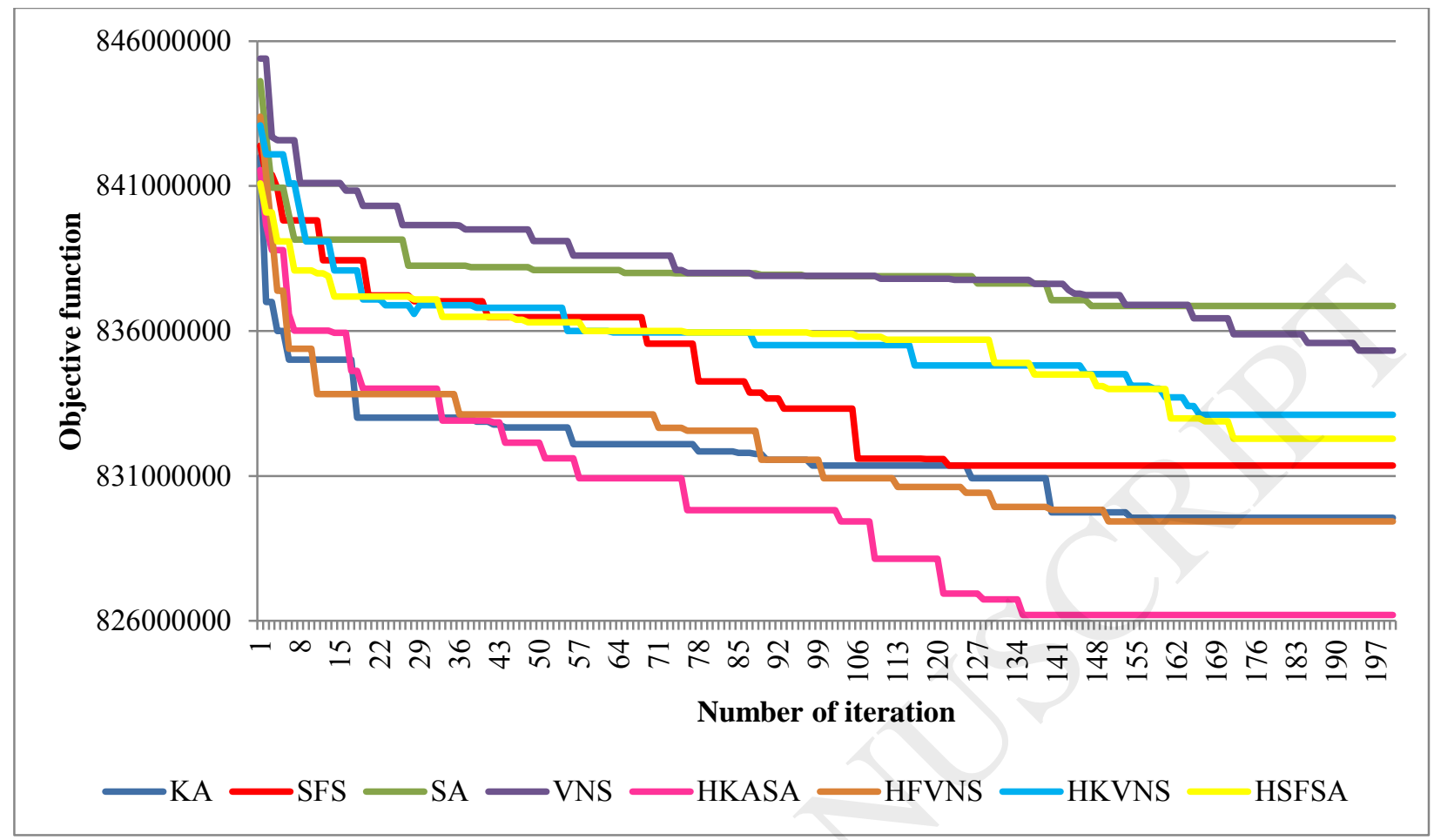

(c)

Figure 13. The behavior of metaheuristics in a same iteration (i.e. (a) for P10, (b) for P16 and (c) for P22) 
Interval Plot of KA; SFS; SA; VNS; HKASA; HFVNS; HKVNS; HSFSA

95\% CI for the Mean

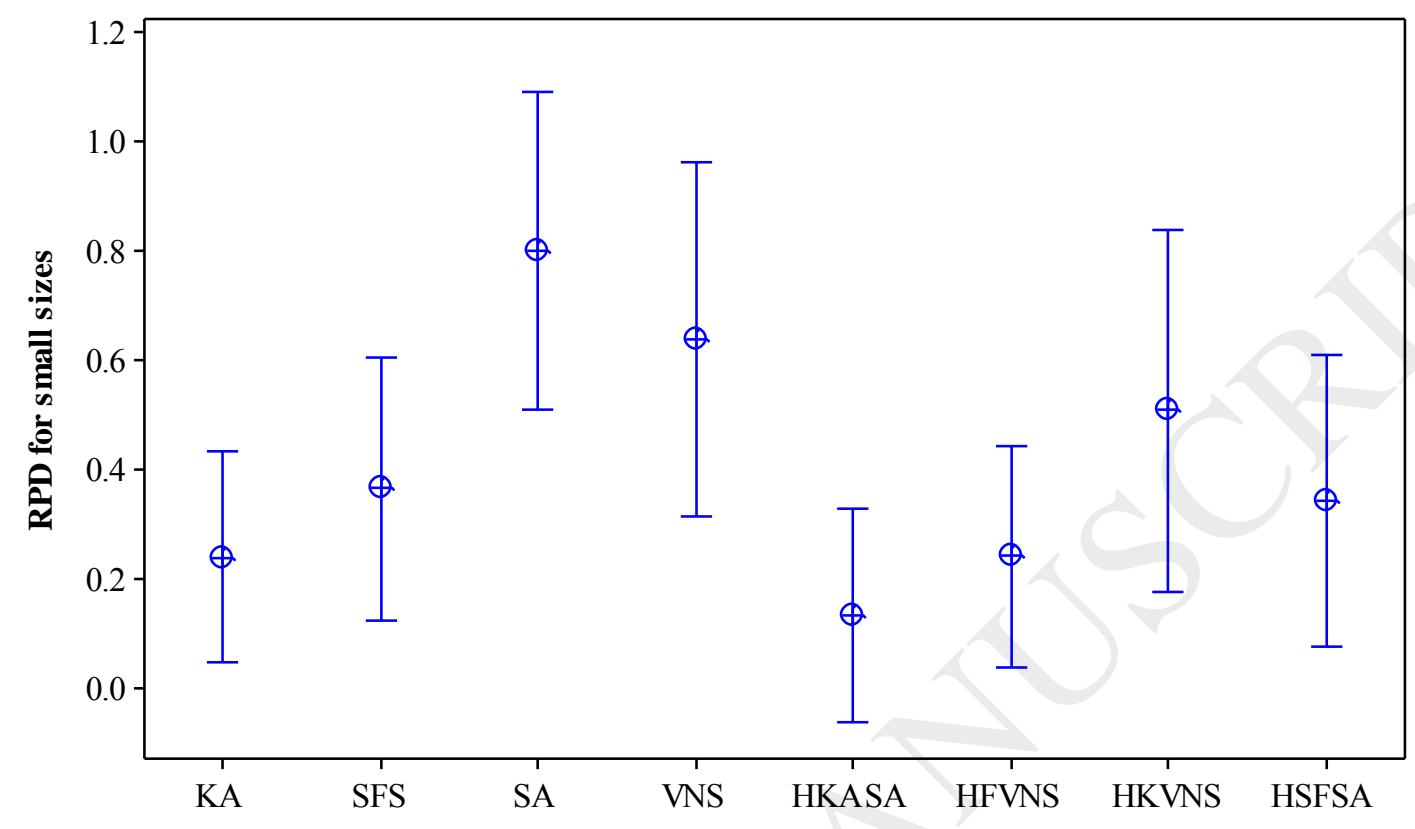

(a)

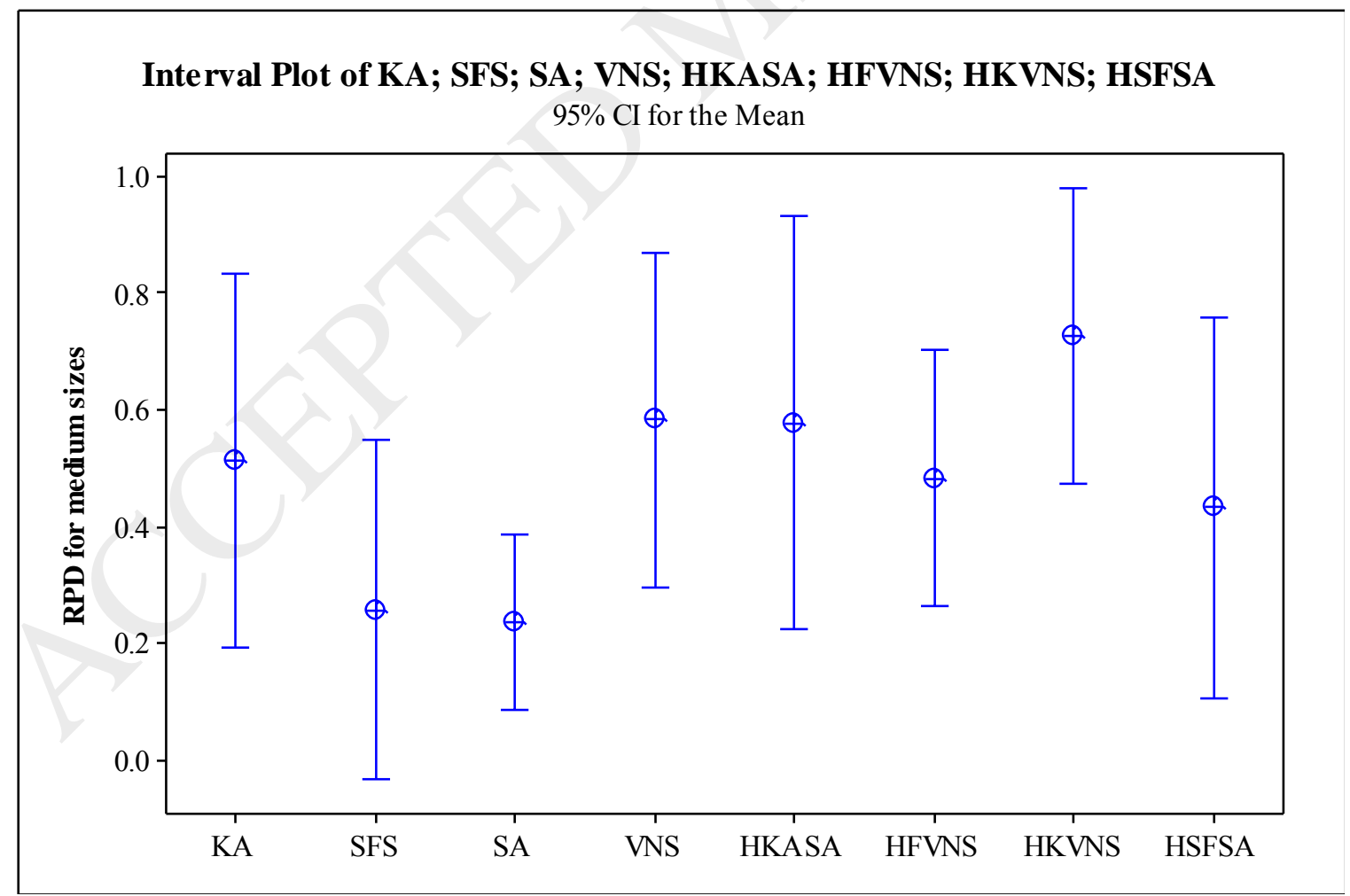

(b) 
Interval Plot of KA; SFS; SA; VNS; HKASA; HFVNS; HKVNS; HSFSA

95\% CI for the Mean

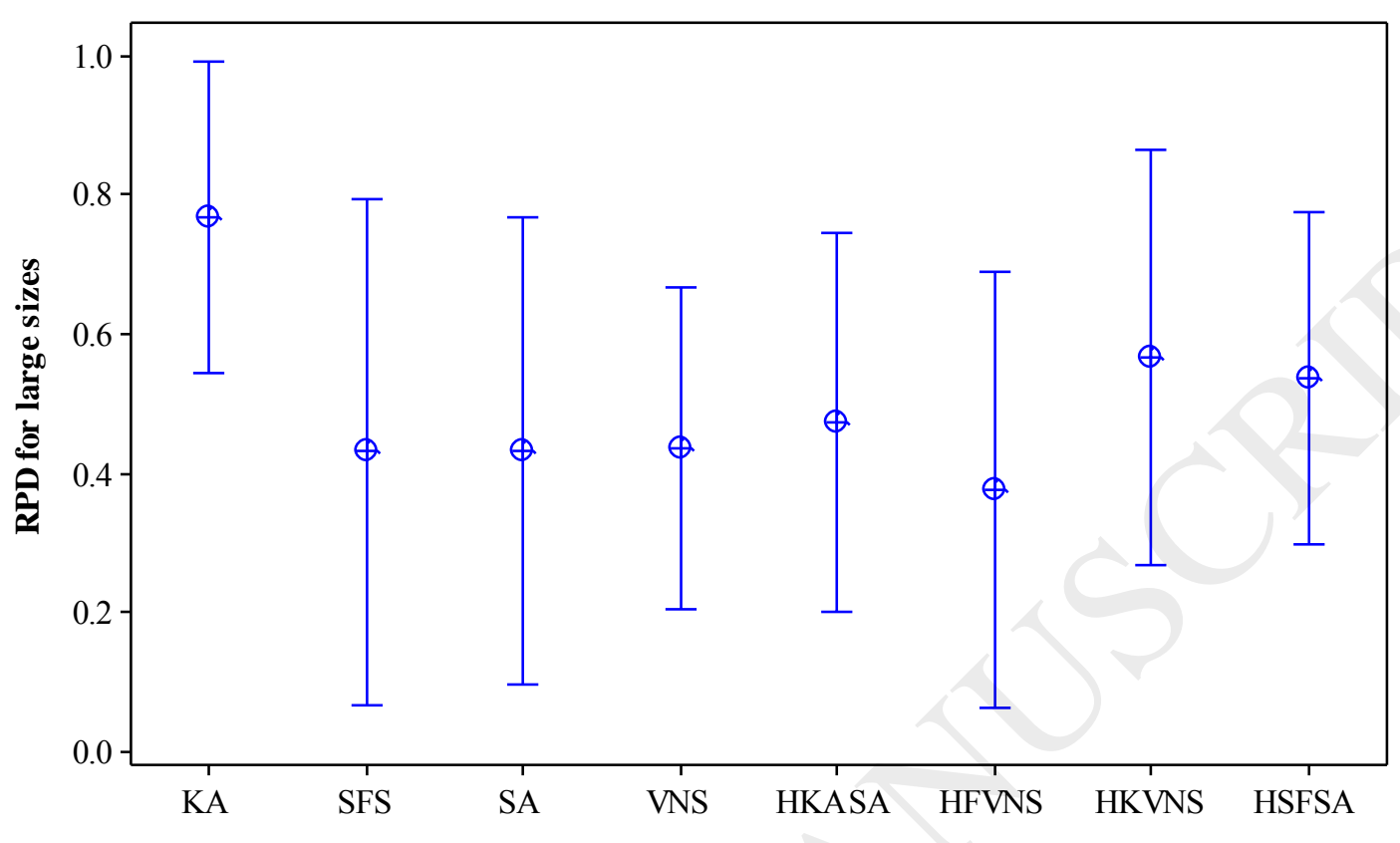

(c)

Figure 14. Means plot and LSD intervals for the presented algorithms (i.e. (a) for small sizes, (b) for medium sizes and (c) for large sizes) 


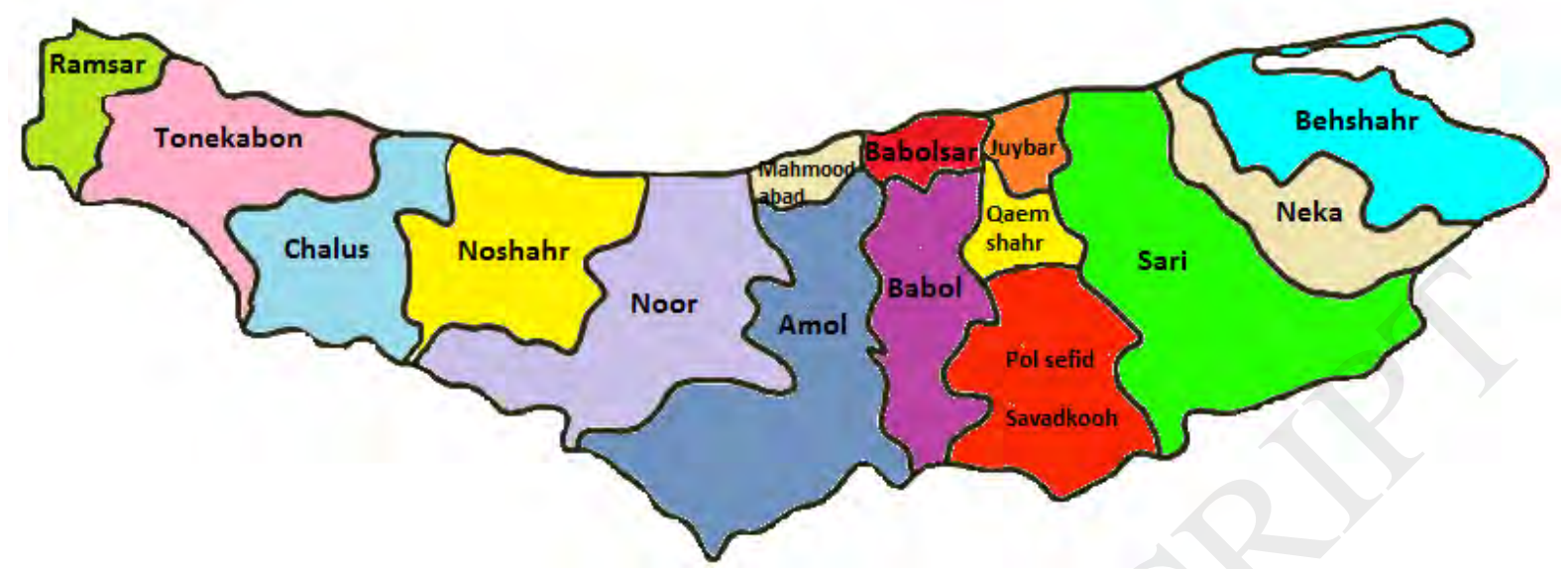

Figure 15. The customer zones in our case study from Mazandaran province [15] 


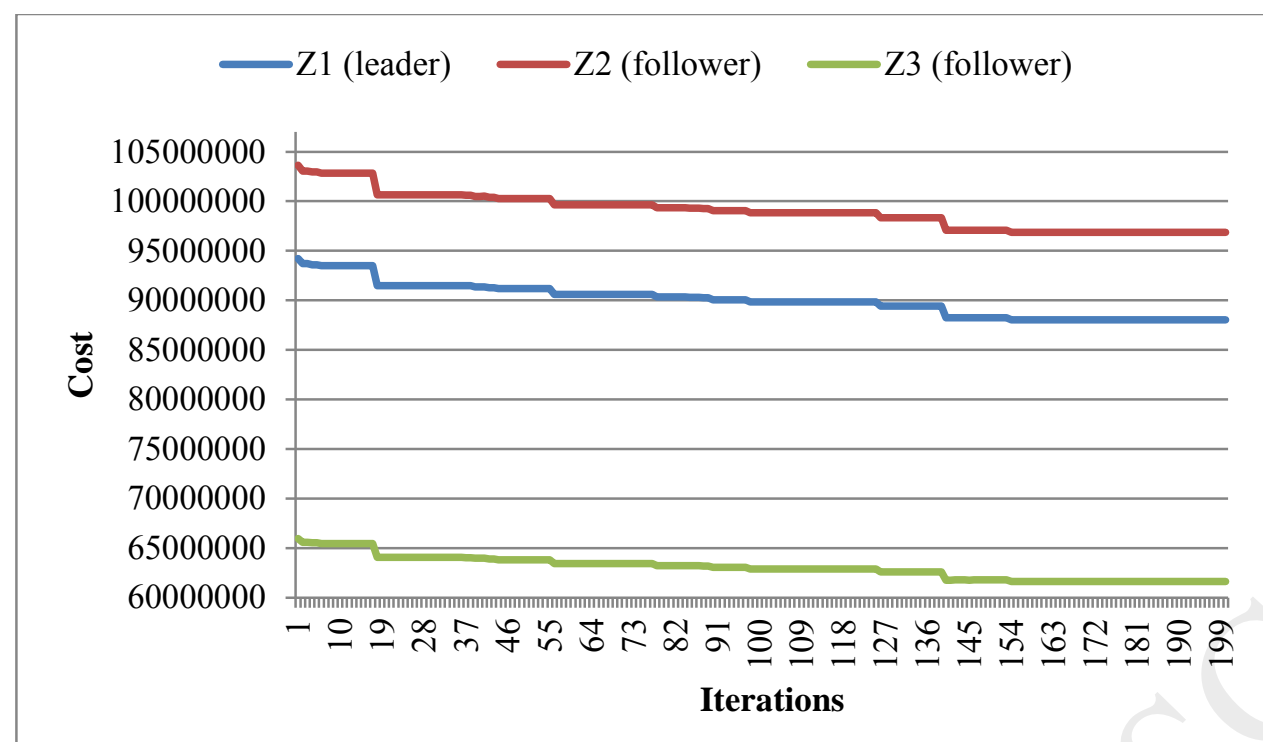

Figure 16. The behavior of decision levels from one run for our case study 


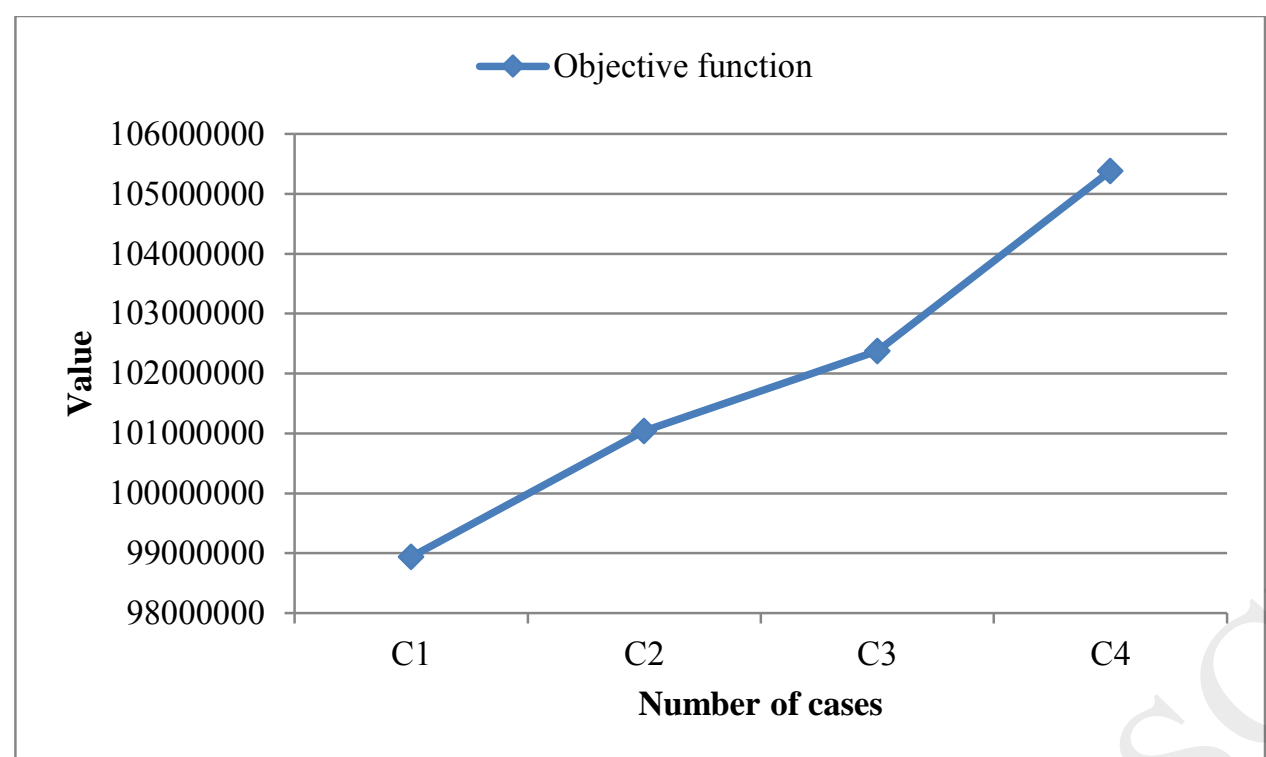

Figure 17. The behavior of objective function 
Table 1. The level, number and size of test problems

\begin{tabular}{|c|c|c|}
\hline The levels of problem & Number of problem $\left(\mathrm{P}_{\mathrm{i}}\right)$ & Size of problems $(I, M, J, L, N, R, P)$ \\
\hline \multirow{8}{*}{ Small } & P1 & $(7,5,10,9,4,3,10)$ \\
\hline & $\mathrm{P} 2$ & $(11,8,12,13,5,4,10)$ \\
\hline & P3 & $(14,12,16,15,8,7,10)$ \\
\hline & $\mathrm{P} 4$ & $(17,16,15,16,11,10,10)$ \\
\hline & P5 & $(19,14,17,19,14,12,10)$ \\
\hline & P6 & $(23,16,21,20,15,13,10)$ \\
\hline & P7 & $(25,29,30,31,19,18,10)$ \\
\hline & P8 & $(27,30,32,32,21,19,10)$ \\
\hline \multirow{8}{*}{ Medium } & P9 & $(34,32,33,35,23,20,10)$ \\
\hline & $\mathrm{P} 10$ & $(37,35,34,37,25,21,10)$ \\
\hline & P11 & $(41,37,36,39,27,22,10)$ \\
\hline & P12 & $(43,39,38,41,29,23,10)$ \\
\hline & P13 & $(45,43,36,39,27,23,10)$ \\
\hline & P14 & $(47,46,38,41,29,24,10)$ \\
\hline & $\mathrm{P} 15$ & $(49,49,40,44,32,26,10)$ \\
\hline & P16 & $(52,44,42,46,32,26,10)$ \\
\hline \multirow{8}{*}{ Large } & P17 & $(67,55,59,111,36,31,10)$ \\
\hline & P18 & $(71,57,61,115,37,32,10)$ \\
\hline & P19 & $(75,59,63,119,39,33,10)$ \\
\hline & $\mathrm{P} 20$ & $(79,61,65,123,40,34,10)$ \\
\hline & $\mathrm{P} 21$ & $(83,63,67,127,42,35,10)$ \\
\hline & $\mathrm{P} 22$ & $(87,65,69,131,43,36,10)$ \\
\hline & $\mathrm{P} 23$ & $(90,69,73,136,46,38,10)$ \\
\hline & $\mathrm{P} 24$ & $(94,72,76,140,49,40,10)$ \\
\hline
\end{tabular}


Table 2. Parameters and their surfaces

\begin{tabular}{|l|l|}
\hline Parameters & Surfaces \\
\hline$P C_{i}^{I}$ & $\operatorname{rand}\{3,4\}$ \\
\hline$P C_{r}^{R}$ & $\operatorname{rand}\{1,2\}$ \\
\hline$P C_{m p}^{M}, P C_{j p}^{J}, P C_{n p}^{N}, P C_{l p}^{L}$ & $\operatorname{rand}\{5,6, \ldots, 10\}$ \\
\hline$M C_{m p}^{M}, M C_{j p}^{J}, M C_{n p}^{N}$ & $\operatorname{rand}\{3,4, \ldots, 8\}$ \\
\hline$T C_{\square}^{\square}$ & $\operatorname{rand}\{3,4, \ldots, 10\}$ \\
\hline$H_{m p}^{M}, H_{j p}^{J}$ & $\operatorname{rand}\{4,5, \ldots, 8\}$ \\
\hline$H_{n p}^{N}$ & $\operatorname{rand}\{2,3, \ldots, 5\}$ \\
\hline$d_{l p}$ & $\sim N(20,4)$ \\
\hline$a_{l p}$ & $\sim N(0.2,0.6)$ \\
\hline
\end{tabular}


Table 3. The list of algorithm's parameters and their levels

\begin{tabular}{|c|c|c|c|c|c|c|}
\hline \multirow{2}{*}{ Algorithms } & \multicolumn{2}{|c|}{ Parameters } & \multicolumn{4}{|l|}{ Levels } \\
\hline & Notation & Description & 1 & 2 & 3 & 4 \\
\hline \multirow{5}{*}{ KA } & MaxIt & $\begin{array}{l}\text { The maximum } \\
\text { number } \\
\text { iteration }\end{array}$ & 200 & 300 & 500 & - \\
\hline & nPop & $\begin{array}{l}\text { The number of } \\
\text { initial population }\end{array}$ & 100 & 150 & 200 & - \\
\hline & PN1 & $\begin{array}{l}\text { The percentage of } \\
\text { N1 Keshtels }\end{array}$ & 0.2 & 0.3 & 0.4 & - \\
\hline & PN2 & $\begin{array}{l}\text { The percentage of } \\
\text { N2 Keshtels }\end{array}$ & 0.1 & 0.2 & 0.3 & - \\
\hline & Smax & $\begin{array}{l}\text { The maximum } \\
\text { number } \\
\text { swirling }\end{array}$ & 2 & 3 & 4 & - \\
\hline \multirow{3}{*}{ SFS } & MaxIt & $\begin{array}{l}\text { The maximum } \\
\text { number } \\
\text { iteration }\end{array}$ & 150 & 200 & 250 & 300 \\
\hline & nPop & $\begin{array}{l}\text { The number of } \\
\text { initial population }\end{array}$ & 100 & 150 & 200 & 250 \\
\hline & MDN & $\begin{array}{l}\text { The maximum } \\
\text { number of } \\
\text { diffusion process }\end{array}$ & 1 & & 3 & 4 \\
\hline \multirow{5}{*}{ SA } & MaxIt & $\begin{array}{l}\text { The maximum } \\
\text { number of } \\
\text { iteration }\end{array}$ & 300 & 500 & 700 & - \\
\hline & SubIt & $\begin{array}{l}\text { The maximum } \\
\text { number of sub- } \\
\text { iteration for sub- } \\
\text { loop }\end{array}$ & 20 & 30 & 50 & - \\
\hline & T0 & Initial temperature & 1000 & 1500 & 2000 & - \\
\hline & Tdamp & $\begin{array}{l}\text { The rate of } \\
\text { reduction } \\
\text { temperature }\end{array}$ & 0.9 & 0.99 & - & - \\
\hline & $\mathrm{Mn}$ & $\begin{array}{l}\text { The procedure of } \\
\text { neighboring }\end{array}$ & Swap & Reversion & Insertion & - \\
\hline \multirow[b]{2}{*}{ VNS } & MaxIt & $\begin{array}{l}\text { The maximum } \\
\text { number } \\
\text { iteration }\end{array}$ & 500 & 800 & - & - \\
\hline & K & $\begin{array}{l}\text { Number of } \\
\text { neighbors shake }\end{array}$ & 1 (i.e. Swap) & $\begin{array}{l}2 \text { (i.e. Swap } \\
\text { and } \\
\text { Reversion) }\end{array}$ & $\begin{array}{l}3 \text { (i.e. Swap, } \\
\text { Reversion } \\
\text { and } \\
\text { Insertion) }\end{array}$ & - \\
\hline \multirow{5}{*}{ HKASA } & MaxIt & $\begin{array}{l}\text { The maximum } \\
\text { number } \\
\text { iteration }\end{array}$ & 200 & 300 & 500 & - \\
\hline & nPop & $\begin{array}{l}\text { The number of } \\
\text { initial population }\end{array}$ & 100 & 150 & 200 & - \\
\hline & PN1 & $\begin{array}{l}\text { The percentage of } \\
\text { N1 Keshtels }\end{array}$ & 0.2 & 0.3 & 0.4 & - \\
\hline & PN2 & $\begin{array}{l}\text { The percentage of } \\
N 2 \text { Keshtels }\end{array}$ & 0.1 & 0.2 & 0.3 & - \\
\hline & Smax & $\begin{array}{lr}\text { The maximum } \\
\text { number }\end{array}$ & 2 & 3 & 4 & - \\
\hline
\end{tabular}




\begin{tabular}{|c|c|c|c|c|c|c|}
\hline & & swirling & & & & \\
\hline & T0 & Initial temperature & 1000 & 1500 & 2000 & - \\
\hline & Tdamp & $\begin{array}{l}\text { The rate of } \\
\text { reduction } \\
\text { temperature }\end{array}$ & 0.9 & 0.99 & - & - \\
\hline \multirow{3}{*}{ HFVNS } & MaxIt & $\begin{array}{l}\text { The maximum } \\
\text { number } \\
\text { iteration }\end{array}$ & 200 & 300 & 500 & - \\
\hline & nPop & $\begin{array}{l}\text { The number of } \\
\text { initial population }\end{array}$ & 100 & 150 & 200 & - \\
\hline & K & $\begin{array}{l}\text { Number of } \\
\text { neighbors shake }\end{array}$ & 1 & 2 & 3 & - \\
\hline \multirow{5}{*}{ HKVNS } & MaxIt & $\begin{array}{l}\text { The maximum } \\
\text { number } \\
\text { iteration }\end{array}$ & 300 & 500 & - & - \\
\hline & nPop & $\begin{array}{l}\text { The number of } \\
\text { initial population }\end{array}$ & 100 & 150 & 200 & - \\
\hline & PN1 & $\begin{array}{l}\text { The percentage of } \\
\text { N1 Keshtels }\end{array}$ & 0.2 & 0.3 & 0.4 & - \\
\hline & Smax & $\begin{array}{l}\text { The maximum } \\
\text { number } \\
\text { swirling }\end{array}$ & 2 & 3 & 4 & - \\
\hline & K & $\begin{array}{l}\text { Number of } \\
\text { neighbors shake }\end{array}$ & 1 & & 3 & - \\
\hline \multirow{5}{*}{ HSFSA } & MaxIt & $\begin{array}{l}\text { The maximum } \\
\text { number } \\
\text { iteration }\end{array}$ & 200 & 300 & 500 & - \\
\hline & nPop & $\begin{array}{l}\text { The number of } \\
\text { initial population }\end{array}$ & 100 & 150 & 200 & - \\
\hline & T0 & Initial temperature & 1000 & 1500 & 2000 & - \\
\hline & Tdamp & $\begin{array}{l}\text { The rate of } \\
\text { reduction } \\
\text { temperature }\end{array}$ & 0.9 & 0.99 & - & - \\
\hline & $\mathrm{Mn}$ & $\begin{array}{l}\text { The procedure of } \\
\text { neighboring }\end{array}$ & Swap & Reversion & Insertion & - \\
\hline
\end{tabular}


Table 4. The tuned parameters of algorithms

\begin{tabular}{|l|l|}
\hline Algorithms & Parameters \\
\hline KA & MaxIt $=500 ; \mathrm{nPop}=200 ; \mathrm{PN} 1=0.2 ; \mathrm{PN} 2=0.2 ; \mathrm{Smax}=3 ;$ \\
\hline SFS & MaxIt $=250 ; \mathrm{nPop}=250 ; \mathrm{MND}=4 ;$ \\
\hline VNS & MaxIt $=800 ; \mathrm{K}=3 ;$ \\
\hline SA & MaxIt $=700 ; \mathrm{SubIt}=30 ; \mathrm{T} 0=2000 ;$ Tdamp $=0.99 ; \mathrm{Mn}=$ Reversion; \\
\hline HKASA & MaxIt $=500 ; \mathrm{nPop}=200 ; \mathrm{PN} 1=0.4 ; \mathrm{PN} 2=0.3 ; \mathrm{Smax}=3 ; \mathrm{T} 0=1500 ; \mathrm{Tdamp}=0.99 ;$ \\
\hline HFVNS & MaxIt $=500 ; \mathrm{nPop}=200 ; \mathrm{K}=3 ;$ \\
\hline HKVNS & MaxIt $=500 ; \mathrm{nPop}=200 ; \mathrm{PN} 1=0.3 ; \mathrm{Smax}=4 ; \mathrm{K}=1 ;$ \\
\hline HSFSA & MaxIt $=500 ; \mathrm{nPop}=150 ; \mathrm{T} 0=1000 ;$ Tdamp $=0.99 ; \mathrm{Mn}=$ Insertion; \\
\hline
\end{tabular}


Table 5. The results of algorithm in small sizes ( $G a p=\frac{Z_{S O L}-Z_{E X}}{Z_{E X}}$, CPU=consuming time, OUT=output of method: the value of the first level objective function; $\mathrm{SD}=$ standard deviation; $\mathrm{HT}=$ hitting time)

\begin{tabular}{|c|c|c|c|c|c|c|c|c|c|}
\hline \multicolumn{2}{|c|}{ Algorithms } & P1 & P2 & P3 & P4 & P5 & P6 & P7 & P8 \\
\hline \multirow{2}{*}{ EX } & OUT & 634 & 38244 & 1786452 & 5178906 & 5869463 & 6755842 & 28331655 & 36482531 \\
\hline & CPU & 344 & 5710 & 151838 & 199466 & 314212 & 500481 & 900208 & 1167884 \\
\hline \multirow{5}{*}{ KA } & OUT & 634 & 40673 & 1943765 & 5466345 & 6794232 & 7054774 & 29937066 & 37865514 \\
\hline & CPU & 478 & 624 & 1167 & 1245 & 1536 & 1672 & 2209 & 3454 \\
\hline & HT & 183 & 385 & 671 & 962 & 1078 & 1174 & 1598 & 2833 \\
\hline & SD & 0 & 0 & 18245.15 & 89457.8 & 158422 & 114726.7 & 568422 & 792164 \\
\hline & Gap & 0 & 0.000006 & 0.088 & 0.0555 & 0.15755 & 0.04424 & 0.05666 & 0.03790 \\
\hline \multirow{5}{*}{ SFS } & OUT & 634 & 40673 & 1964738 & 5478251 & 6944536 & 7068346 & 30157462 & 38316547 \\
\hline & CPU & 588 & 661 & 1354 & 1467 & 1726 & 1933 & 2467 & 3987 \\
\hline & HT & 394 & 513 & 1065 & 1189 & 1425 & 1367 & 1825 & 2032 \\
\hline & SD & 0 & 0 & 21476.92 & 94185.1 & 170647 & 121447.6 & 682576 & 658934 \\
\hline & Gap & 0 & 0.000006 & 0.0997 & 0.0578 & 0.1831 & 0.0462 & 0.0644 & 0.05027 \\
\hline \multirow{5}{*}{ SA } & OUT & 634 & 40673 & 1985322 & 5567329 & 7355678 & 7158953 & 33648752 & 38578475 \\
\hline & CPU & 486 & 521 & 1041 & 1135 & 1491 & 1582 & 2145 & 3014 \\
\hline & HT & 218 & 362 & 653 & 715 & 1065 & 1264 & 1873 & 2166 \\
\hline & SD & 0 & 792.97 & 27183.53 & 106745.3 & 213687 & 123859.3 & 822143 & 813562 \\
\hline & Gap & 0 & 0.000006 & 0.1113 & 0.0750 & 0.2532 & 0.0596 & 0.18767 & 0.05745 \\
\hline \multirow{5}{*}{ VNS } & OUT & 634 & 40673 & 1964351 & 5538914 & 6955232 & 7144398 & 32893651 & 38169542 \\
\hline & CPU & 435 & 563 & 978 & 1092 & 1323 & 1410 & 1915 & 2968 \\
\hline & HT & 219 & 438 & 512 & 854 & 715 & 821 & 1327 & 2491 \\
\hline & SD & 0 & 842.55 & 19867.43 & 97435.2 & 215756.2 & 112633.7 & 748521 & 977834 \\
\hline & Gap & 0 & 0.000006 & 0.0995 & 0.0695 & 0.1849 & 0.05751 & 0.16102 & 0.04624 \\
\hline \multirow{5}{*}{ HKASA } & OUT & 634 & 39822 & 1883702 & 5438925 & 6432913 & 6852742 & 30648220 & 38647158 \\
\hline & CPU & 514 & 695 & 1206 & 1398 & 1753 & 2371 & 2541 & 4012 \\
\hline & HT & 187 & 431 & 725 & 892 & 1327 & 1954 & 1926 & 2841 \\
\hline & SD & 0 & 538.28 & 16542.85 & 76345.2 & 165473 & 106554.7 & 437819 & 674821 \\
\hline & Gap & 0 & 0.000004 & 0.0544 & 0.0502 & 0.0959 & 0.01434 & 0.0817 & 0.05933 \\
\hline \multirow{5}{*}{ HFVNS } & OUT & 634 & 40673 & 1932766 & 5647232 & 6748932 & 7066543 & 29145692 & 38557169 \\
\hline & CPU & 612 & 645 & 1287 & 1389 & 1673 & 2185 & 2317 & 3503 \\
\hline & HT & 348 & 517 & 736 & 819 & 1053 & 1582 & 1825 & 2361 \\
\hline & SD & 0 & 0 & 18542.38 & 84291.5 & 159547 & 119856.3 & 619054 & 684923 \\
\hline & Gap & 0 & 0 & 0.0819 & 0.0904 & 0.1498 & 0.04598 & 0.02873 & 0.05686 \\
\hline \multirow{5}{*}{ HKVNS } & OUT & 634 & 40673 & 1925804 & 5366910 & 6533184 & 7183295 & 29668523 & 38973615 \\
\hline & CPU & 504 & 536 & 1187 & 1272 & 1416 & 1978 & 2467 & 3683 \\
\hline & HT & 407 & 475 & 874 & 1156 & 1285 & 1543 & 2185 & 2763 \\
\hline & SD & 0 & 729.31 & 25317.89 & 96438.1 & 186477 & 126558.4 & 522614 & 538511 \\
\hline & Gap & 0 & 0.000006 & 0.0780 & 0.03630 & 0.11308 & 0.06327 & 0.04718 & 0.06828 \\
\hline \multirow{5}{*}{ HSFSA } & OUT & 634 & 40673 & 1983314 & 5428129 & 6658316 & 7216554 & 29872436 & 38591173 \\
\hline & CPU & 518 & 548 & 1275 & 1465 & 1617 & 2034 & 2573 & 3874 \\
\hline & HT & 321 & 363 & 897 & 1043 & 1285 & 1753 & 2174 & 2896 \\
\hline & SD & 0 & 0 & 21635.82 & 102572.3 & 174393 & 119949.1 & 589156 & 562897 \\
\hline & Gap & 0 & 0.000006 & 0.1101 & 0.0481 & 0.1343 & 0.0681 & 0.05438 & 0.05779 \\
\hline
\end{tabular}


Table 6. The results of algorithms in medium sizes ( $\mathrm{B}=$ best, $\mathrm{W}=$ worst, $\mathrm{M}=$ mean).

\begin{tabular}{|c|c|c|c|c|c|c|c|c|c|}
\hline \multicolumn{2}{|c|}{ Algorithms } & P9 & P10 & P11 & P12 & P13 & P14 & P15 & P16 \\
\hline \multirow{6}{*}{ KA } & B & 47368194 & 56379218 & 71560375 & 86794355 & 78660852 & 95490322 & 115750493 & 130508422 \\
\hline & $\mathrm{W}$ & 49378215 & 60229514 & 73280513 & 87942506 & 81550824 & 98340218 & 121618765 & 132509318 \\
\hline & $\mathrm{M}$ & 48142306 & 57852939 & 72192465 & 87070409 & 79424280 & 96215857 & 117940134 & 131134144 \\
\hline & SD & 858935.3 & 1621788 & 672669.4 & 388419.3 & 976841.2 & 1095466 & 2234100 & 747366 \\
\hline & $\mathrm{CPU}$ & 2854 & 4167 & 5188 & 5573 & 7367 & 8254 & 8913 & 9435 \\
\hline & HT & 1933 & 3451 & 3719 & 3166 & 5097 & 6518 & 6732 & 7180 \\
\hline \multirow{6}{*}{ SFS } & $\mathrm{B}$ & 47590134 & 57228415 & 70842275 & 87052788 & 79578622 & 95731804 & 116854903 & 131043626 \\
\hline & $\mathrm{W}$ & 50178433 & 60189455 & 72843902 & 88100436 & 80762294 & 98478322 & 122207685 & 133021788 \\
\hline & $\mathrm{M}$ & 48640002 & 58461998 & 71772803 & 87486113 & 80074134 & 96657361 & 118492111 & 131736927 \\
\hline & SD & 873232.7 & 901495.5 & 713596.9 & 373102 & 489590.3 & 1062298 & 1848624 & 728826.23 \\
\hline & CPU & 3214 & 4586 & 5186 & 5893 & 7519 & 8322 & 9150 & 9734 \\
\hline & HT & 1895 & 3893 & 4275 & 4285 & 6053 & 6218 & 7502 & 8187 \\
\hline \multirow{6}{*}{ SA } & $\mathrm{B}$ & 48726155 & 58402518 & 70582904 & 87390214 & 79632508 & 96570352 & 116743512 & 131390527 \\
\hline & $\mathrm{W}$ & 50614892 & 61304276 & 72168846 & 88570425 & 81550934 & 98567430 & 122478303 & 134025783 \\
\hline & $\mathrm{M}$ & 49641341 & 59805057 & 71376880 & 87863302 & 80605331 & 97358185 & 119362223 & 132592496 \\
\hline & SD & 707933.03 & 1045779.2 & 510833 & 427702.7 & 755394.7 & 792318.7 & 1999902 & 922030.1 \\
\hline & CPU & 2567 & 3185 & 4507 & 5109 & 5722 & 6488 & 7913 & 8452 \\
\hline & HT & 1627 & 2852 & 3182 & 4278 & 3186 & 5265 & 6054 & 7278 \\
\hline \multirow{6}{*}{ VNS } & $\mathrm{B}$ & 48270125 & 59350216 & 70038172 & 87043722 & 80550628 & 96053813 & 116589413 & 131390428 \\
\hline & $\mathrm{W}$ & 51490266 & 61447328 & 72548942 & 88754013 & 82753406 & 98475518 & 122358725 & 135108549 \\
\hline & $\mathrm{M}$ & 49473396 & 60296901 & 71179714 & 87833574 & 81565248 & 96975120 & 118809951 & 133153442 \\
\hline & SD & 1056406 & 732496.4 & 863434.54 & 560975.4 & 1038149 & 888058 & 2302676 & 1321931.4 \\
\hline & $\mathrm{CPU}$ & 2426 & 3379 & 4334 & 4897 & 6276 & 6921 & 8254 & 8543 \\
\hline & HT & 1865 & 2519 & 2953 & 3865 & 5732 & 5367 & 6243 & 7158 \\
\hline \multirow{6}{*}{ HKASA } & B & 47863721 & 56331852 & 69483326 & 86590423 & 78642506 & 95137589 & 115026734 & 129632860 \\
\hline & $\mathrm{W}$ & 49032564 & 59448215 & 72842248 & 87590484 & 81095632 & 97861352 & 121675532 & 133201784 \\
\hline & M & 48150967 & 57799686 & 70466975 & 86829144 & 79346786 & 95824804 & 115913240 & 130553624 \\
\hline & SD & 386221.2 & 1189708.8 & 1293214.3 & 358938 & 1052124 & 1085812 & 2298794 & 1262423.1 \\
\hline & $\mathrm{CPU}$ & 3058 & 4367 & 5392 & 5813 & 7516 & 8461 & 9158 & 9671 \\
\hline & HT & 2275 & 3681 & 3913 & 4103 & 4613 & 5921 & 5825 & 6135 \\
\hline \multirow{6}{*}{ HFVNS } & $\mathrm{B}$ & 47489322 & 57115268 & 70158634 & 87032517 & 78849056 & 96314508 & 116032584 & 130285902 \\
\hline & $\mathrm{W}$ & 50226317 & 59842612 & 72660822 & 87990534 & 80972431 & 98678503 & 122301982 & 134724816 \\
\hline & $\mathrm{M}$ & 48405293 & 58172007 & 71006254 & 87409059 & 79588606 & 97256686 & 118455486 & 130581830 \\
\hline & SD & 1028300.4 & 1020797.1 & 1082268 & 375213.9 & 746725 & 911431.3 & 2330497.1 & 1126188.5 \\
\hline & CPU & 2820 & 3982 & 4760 & 5395 & 6897 & 7621 & 8702 & 9138 \\
\hline & HT & 1765 & 2319 & 2942 & 3816 & 4275 & 6174 & 7217 & 8259 \\
\hline \multirow{6}{*}{ HKVNS } & B & 47829133 & 57139204 & 70526608 & 86890435 & 78905422 & 96140329 & 115490326 & 130195672 \\
\hline & W & 50367288 & 60278845 & 73048625 & 88570423 & 80558794 & 98674210 & 122843702 & 133569436 \\
\hline & M & 48837783 & 58342723 & 71656097 & 87421956 & 79457267 & 97365555 & 117795425 & 131448493 \\
\hline & SD & 1091983 & 1201047 & 838710 & 655169.3 & 684407.9 & 970813 & 2905090.6 & 1426765.3 \\
\hline & $\mathrm{CPU}$ & 2640 & 3773 & 4761 & 5235 & 6821.5 & 7587.5 & 8583.5 & 8989 \\
\hline & HT & 1654 & 2418 & 2914 & 3627 & 5031 & 5389 & 5924 & 7143 \\
\hline \multirow{6}{*}{ HSFSA } & B & 48631255 & 57042566 & 70526642 & 86559302 & 79536728 & 96150438 & 116480322 & 130926675 \\
\hline & W & 50279132 & 61594285 & 72446708 & 87460366 & 81042604 & 98879430 & 121468304 & 134226804 \\
\hline & $\mathrm{M}$ & 49171366 & 59191277 & 71076788 & 86938075 & 80136360 & 97386938 & 118593911 & 132076960 \\
\hline & SD & 694189.1 & 1764377 & 749320.9 & 357762.3 & 565234.7 & 1052370 & 1840957.7 & 1233427.6 \\
\hline & $\mathrm{CPU}$ & 2890 & 3885 & 4846 & 5501 & 6620 & 7405 & 8531 & 9093 \\
\hline & HT & 1763 & 2910 & 3473 & 4037 & 4852 & 5281 & 6764 & 6419 \\
\hline
\end{tabular}


Table 7. The results of algorithms in large sizes.

\begin{tabular}{|c|c|c|c|c|c|c|c|c|c|}
\hline \multicolumn{2}{|c|}{ Algorithms } & P17 & P18 & P19 & P20 & P21 & P22 & P23 & P24 \\
\hline \multirow{6}{*}{ KA } & B & 453892142 & 535894024 & 619736844 & 654823889 & 682480972 & 825687902 & 931859302 & 1018953622 \\
\hline & $\mathrm{W}$ & 464950340 & 556702542 & 621746204 & 673920135 & 692159425 & 840313293 & 964499223 & 1135435470 \\
\hline & $\mathrm{M}$ & 457735206 & 541201591 & 620555159 & 660000459 & 684563377 & 829558417 & 943765304 & 1053949835 \\
\hline & SD & 4194385 & 8373931 & 738196.4 & 7641154.16 & 3276387.3 & 5852345.5 & 13183943.5 & 41711061.1 \\
\hline & CPU & 12832 & 13647 & 14843 & 15726 & 17218 & 18374 & 19681 & 20164 \\
\hline & HT & 8903 & 9714 & 10251 & 10897 & 13428 & 13892 & 15425 & 16829 \\
\hline \multirow{6}{*}{ SFS } & B & 461095832 & 538871290 & 619593204 & 655270188 & 681780444 & 826785992 & 941859642 & 1085490322 \\
\hline & W & 467328102 & 553491204 & 621835266 & 663809145 & 698745223 & 841465486 & 965594376 & 1185370268 \\
\hline & M & 463336236 & 544429155 & 620652030 & 658792692 & 688825291 & 831358449 & 949440137 & 1128661610 \\
\hline & SD & 2298599.7 & 5704208.5 & 784172.6 & 3452651.93 & 6312213.35 & 5535731.13 & 7057310.18 & 37970564.4 \\
\hline & CPU & 13574 & 14243 & 16375 & 17205 & 18154 & 19632 & 21054 & 22417 \\
\hline & HT & 9743 & 10326 & 10843 & 10725 & 11439 & 12754 & 12923 & 14062 \\
\hline \multirow{6}{*}{ SA } & B & 462504631 & 542042566 & 620167842 & 652901675 & 685326798 & 827516820 & 943026842 & 1096585240 \\
\hline & W & 470148253 & 560327114 & 622186437 & 673902194 & 697325418 & 849210456 & 962759042 & 1150932744 \\
\hline & $\mathrm{M}$ & 466089111 & 549982210 & 621018007 & 663077770 & 690788869 & 838864723 & 950932829 & 1123673887 \\
\hline & SD & 2529774.6 & 6148538.6 & 772273.84 & 6528897 & 3804185.66 & 7258949.57 & 6103689.29 & 19169868.8 \\
\hline & CPU & 10732 & 11854 & 12106 & 12548 & 13287 & 14065 & 14622 & 15392 \\
\hline & HT & 7435 & 9215 & 10632 & 11433 & 9784 & 10822 & 11437 & 11829 \\
\hline \multirow{6}{*}{ VNS } & B & 461794832 & 540867542 & 619832487 & 653189530 & 684269825 & 826439024 & 943872164 & 1082473912 \\
\hline & W & 469382174 & 558319306 & 621874953 & 668429315 & 697452810 & 846527910 & 970328492 & 1162385904 \\
\hline & $\mathrm{M}$ & 465210359 & 549103295 & 620740654 & 660377064 & 684709258 & 835330990 & 955941856 & 1122349483 \\
\hline & SD & 2687790 & 6505083.8 & 767769.206 & 4914794.78 & 2406872.75 & 6833056.8 & 8713729.53 & 26787737.5 \\
\hline & CPU & 10365 & 10854 & 11437 & 12679 & 12935 & 13209 & 14825 & 15632 \\
\hline & HT & 7934 & 9452 & 10268 & 11539 & 12036 & 12847 & 13092 & 13627 \\
\hline \multirow{6}{*}{ HKASA } & B & 453672186 & 532564372 & 619206145 & 647390216 & 678324678 & 821054362 & 931705462 & 1022634788 \\
\hline & $\mathrm{W}$ & 468726170 & 551674386 & 621186432 & 661458795 & 682214582 & 839437184 & 953820912 & 1118423906 \\
\hline & $\mathrm{M}$ & 457078470 & 538372040 & 619794448 & 650064553 & 679164679 & 826199891 & 936446709 & 1042585566 \\
\hline & SD & 5051986.3 & 6759865.9 & 700824.823 & 4478698.74 & 1282530.5 & 7530075.4 & 7433018.33 & 34108027.9 \\
\hline & CPU & 13627 & 14296 & 15621 & 16328 & 18513 & 20173 & 22537 & 24072 \\
\hline & HT & 8977 & 10534 & 11762 & 11351 & 13275 & 12809 & 14185 & 15311 \\
\hline \multirow{6}{*}{ HFVNS } & B & 460864238 & 541906543 & 619428614 & 648239015 & 682453167 & 825648932 & 935136742 & 1031278430 \\
\hline & $\mathrm{W}$ & 467204652 & 558214350 & 621426082 & 662451955 & 698854236 & 837218455 & 954678193 & 1137823091 \\
\hline & $\mathrm{M}$ & 461286932 & 547561265 & 619916723 & 652507392 & 688681808 & 829430003 & 942658941 & 1065791640 \\
\hline & SD & 1608614.5 & 5857257.9 & 681089.65 & 5622396.23 & 5720400.13 & 4178682 & 6871135.99 & 38335259.2 \\
\hline & CPU & 12290 & 12887 & 14399 & 15394 & 16066 & 17062 & 18562 & 19703 \\
\hline & HT & 10483 & 11928 & 12702 & 12835 & 13849 & 12532 & 13674 & 15263 \\
\hline \multirow{6}{*}{ HKVNS } & B & 451209552 & 540427482 & 620642188 & 650325167 & 684536226 & 827750941 & 932768492 & 1025643819 \\
\hline & $\mathrm{W}$ & 470251869 & 560319472 & 621972416 & 666843628 & 697426044 & 842206462 & 953682172 & 1162478390 \\
\hline & $\mathrm{M}$ & 459875183 & 547595993 & 621221304 & 655177948 & 687501947 & 833110661 & 941317345 & 1066990964 \\
\hline & SD & 7162596.6 & 7736031.6 & 486577.81 & 5492779.14 & 4514986.52 & 5521878 & 7698611.13 & 43246864.9 \\
\hline & $\mathrm{CPU}$ & 11351 & 11971 & 12799 & 13897 & 14648 & 15275 & 16767 & 17444 \\
\hline & HT & 8533 & 9521 & 10186 & 10794 & 11295 & 12378 & 13026 & 14276 \\
\hline \multirow{6}{*}{ HSFSA } & B & 458765489 & 537248391 & 619527046 & 651489322 & 682241560 & 826437024 & 934803286 & 1012436285 \\
\hline & $\mathrm{W}$ & 469743261 & 553802164 & 621488362 & 669432784 & 697342562 & 842561046 & 953872902 & 1128634166 \\
\hline & M & 462643992 & 544150653 & 620311861 & 655354379 & 687427904 & 832287260 & 941362289 & 1050184802 \\
\hline & SD & 3771856.2 & 6353398.4 & 732277.567 & 5781200.36 & 5314422.55 & 5898405.41 & 6724905.94 & 40095779.6 \\
\hline & $\mathrm{CPU}$ & 11746 & 13286 & 14563 & 15295 & 15821 & 16795 & 17349 & 19386 \\
\hline & HT & 8725 & 9511 & 12437 & 8765 & 14676 & 13253 & 14206 & 16811 \\
\hline
\end{tabular}


Table 8. The sensitivity analyses on the price of raw materials

\begin{tabular}{|l|l|l|}
\hline Number of cases & $\left(P C_{i}, P C_{r}\right)$ & Objective function $\left(\mathrm{Z}_{1}\right)$ \\
\hline $\mathrm{C} 1$ & $(4,1)$ & 98938525 \\
\hline $\mathrm{C} 2$ & $(4,2)$ & 101043946 \\
\hline $\mathrm{C} 3$ & $(4,3)$ & 102378254 \\
\hline $\mathrm{C} 4$ & $(4,4)$ & 105380241 \\
\hline
\end{tabular}

